\title{
Volcanic Ash Region Path Planning Based on Improved A-Star Algorithm
}

\author{
Lan Ma $\mathbb{D}^{1},{ }^{1}$ Haotong Zhang $\mathbb{D}^{1},{ }^{1}$ Shijun Meng $\mathbb{D}^{2},{ }^{2}$ and Jiayue Liu $\mathbb{D D}^{1}$ \\ ${ }^{1}$ School of Air Traffic Management, Civil Aviation University of China, Tianjin 300300, China \\ ${ }^{2}$ School of Electronic Information \& Automation, Civil Aviation University of China, Tianjin 300300, China \\ Correspondence should be addressed to Lan Ma; malan66@263.net
}

Received 3 March 2021; Revised 17 January 2022; Accepted 3 February 2022; Published 28 February 2022

Academic Editor: Giuseppe Musolino

Copyright (C) 2022 Lan Ma et al. This is an open access article distributed under the Creative Commons Attribution License, which permits unrestricted use, distribution, and reproduction in any medium, provided the original work is properly cited.

In civil aviation flight path planning, in order to effectively reduce the safety threat caused by the volcanic ash area to the civil aviation flight, factors such as the speed and acceleration of the aircraft in the volcanic ash area must be considered. In this paper, we propose an improved A-star algorithm by adopting the concept of potential collision set and using the velocity vector idea of optimal reciprocal collision avoidance (ORCA). The improved A-star algorithm selects the optimal speed range of the aircraft from the limited elements, obtains the speed and acceleration set of the aircraft in the volcanic ash area, calculates the flight path through the displacement increment, continuously refreshes the global starting point to the target point, and finally obtains the final path planning results by calculating the total cost value. The improved A-star algorithm is used to plan two flight paths from Madrid to Cairo and Algiers to Rome in volcanic ash areas. The verification results show that the improved A-star algorithm optimizes the flight path planning in the volcanic ash area and has the advantages of less search nodes, a small search range, and short computing time.

\section{Introduction}

Volcanic ash produced by volcanic eruptions will have a serious impact on the vicinity, especially if the volcanic ash cloud rises to the top of the troposphere and above, it will pose a major threat to aviation safety [1]. When a volcano erupts, the ash rises and stays in the atmosphere for a long time. After the ascent tends to stop, the volcanic ash will be affected by the wind to spread and transfer, and aircraft engines flying in the volcanic ash area easily inhale it. The temperature of the engine combustion chamber of modern aircraft is much higher than the melting point of the main component of volcanic ash, which will melt after being inhaled by the engine. As the temperature of high-temperature gas discharged backward drops, the volcanic ash is very easy to recondensate in the engine structure, causing the engine to surge or even stall. There have been flights that have stopped in the air after straying into the volcanic ash region. In order to prevent accidents, one aircraft took 16 minutes to restart the engine after taxiing to 3000 meters, but the fuselage was still severely damaged. In addition to affecting the engine, volcanic ash can also scratch the fuselage surface, affecting the windshield, windows on both sides of the fuselage, the wing surface, the flap, the Fin stabilizer, and even the Pitot tube and the electrostatic probe.

Over the past 20 years, there have been more than 90 records of direct damage to international flights caused by volcanic ash. Recent incidents where volcanic ash has affected flights are as follows. In 2010, a volcanic eruption in Iceland affected flights over Europe and the Atlantic Ocean for more than 20 days. In 2014, due to the eruption of a volcano in Indonesia, several flights were forced to the ground. The Alaska Peninsula continued to erupt in 2016, forcing more and more flights to be canceled. In 2018, a volcanic eruption in Bali caused several flights to be temporarily canceled.

Because the ash particles are less than millimeters in diameter and contain less water of their own, they are undetectable by airborne radar, and it is difficult for pilots to determine with the naked eye. The only thing that can be 
distinguished after straying into the volcanic ash area is that the aircraft fuselage will be surrounded by a hazy glow. The airplane rubs against the particles in the volcanic ash during high-speed flight, generating static electricity. When this phenomenon is discovered, the crew should turn 180 degrees and leave the area as soon as possible. It is more appropriate to reroute around these areas by judging where the ash appears and where it is likely to spread and transfer when affected by high-altitude winds. Our country has not yet been affected by the volcanic eruption, but our flights to Iceland, the United States and Australia, and other paths have been affected by volcanic ash. WAFS has the function of forecasting volcanic ash and other important weather, which can ensure flight safety to a certain extent.

The problem studied in this paper is how to find the optimal path for the aircraft to fly around the ash cloud. At present, the existing methods include traditional classical algorithm (Dijkstra algorithm, artificial potential field method, etc.) and modern intelligent algorithm (A-star algorithm, genetic algorithm, particle swarm optimization, etc.), but the classical algorithm is affected by the setting of conditions, and intelligent algorithms often have the problems with local optimization or high complexity. Therefore, this paper adopts the ORCA algorithm to improve the idea of the A-star algorithm to achieve global and local optimal solutions and reduce complexity.

The main contributions of this paper are as follows:

First, in static path planning, an improved A-star algorithm based on the vector ideas of the optimal reciprocal collision avoidance (ORCA) algorithm with the critical velocity vector as the search boundary is proposed, making the exploration direction no longer restricted by the grid points, shortening the path length, reducing the turning angle, and reducing the distance away from the course. The A-star algorithm grid exploration is simplified to the node exploration in the network diagram, which greatly reduces the number of exploration nodes and the direction of exploration. Thereby the improved algorithm greatly reduces the number of exploration nodes and the operation time of the A-star algorithm; that is, it reduces the complexity while achieving global and local optimal solutions.

Second, the improved A-star algorithm was used to optimize the two paths influenced by volcanic ash from Madrid to Cairo and Algiers to Rome individually. The path planning in the volcanic ash area is better realized. The optimized path is compared and analyzed with the optimization results of other algorithms according to the flight distance, the amount of angle change, run time, and the computation complexity.

The organizational structure of the rest part of the paper is as follows. The second section mainly introduces the related research at home and abroad and analyzes the advantages and disadvantages of different algorithms. In the third section, the algorithm model of volcanic ash area path planning and the improved A-star algorithm are proposed.
The fourth section simulates the improved algorithm and analyzes the simulation results of the two paths covered by volcanic ash from Madrid to Cairo and Algiers to Rome. The fifth section summarizes the full-text work as well as the future research directions.

\section{Related Work}

In the current path planning algorithm, the algorithms are generally divided into two categories according to the obstacle movement forms and the surrounding environments, global path planning, and local path planning. Global path planning refers to the use of algorithms to search path in a state where the surrounding environment of the aircraft is completely known. Generally, there are two steps of map establishment and path search. However, when the surrounding changes suddenly, the path planning is easy to fall into the local optimal solution. It is a preplanning algorithm.

Local path planning refers to the path exploration of the aircraft when the surrounding information is completely unknown or only a small amount of information is known during the flight. Local path planning focuses on the processing of current local information so that the aircraft can obtain better real-time obstacle avoidance capabilities. At the same time, the position and geometric properties of the object are dynamically calibrated in real time. The local path planning combines the modeling of the environment around the aircraft with the search for the optimal path. It requires that the algorithm must have high-speed calculation efficiency and be able to feedback and correct obstacles and the planned path in real time [2].

In the aspect of path planning, Myers et al. proposed an improved Dijkstra algorithm model based on the consideration of aircraft performance to realize the dynamic redistribution of nodes [3]. Three assumptions are added to the mathematical model of multiobjective dynamic resource management to improve the accuracy of dynamic path planning. Cheung proposed a node-based routing algorithm, which introduced the A-star algorithm with the constraints of the best time and the lowest cost [4]. It not only maintains the low complexity of the algorithm but also limits the exploration nodes of the algorithm and improves the efficiency of the algorithm. Patle et al. proposed a path planning algorithm based on probability and fuzzy logic [5]. Probability determines the next move path and real-time planning. This is an improvement to the traditional path planning algorithm, but it is easy to fall into the result of the local optimal solution because of the probability problem. D'Amato et al. used a method based on visibility reduction graph (RVG) to minimize the path length by connecting the nodes of arcs and segments and find the shortest path and optimal path points for different paths. In the preprocessing stage, the space is divided into view areas, and the spatial index is defined as straight lines and curves [6].

Zhang et al. established a multiobjective rerouting planning model in this study for terminal airspace under convective weather conditions in the tactical air traffic flow 
management phase [7]. Although the meteorological factors were objectively considered and the model was established, the selection of the model's influencing factors will have a greater impact on the results, and careful selection is required. The computation complexity of nondominated sorting is reduced, the sampling space is enlarged, and the crowding degree and crowding degree operators are introduced to make up for the shortcomings of the traditional genetic algorithm. Li also studied scattered hazardous weather, proposed a specific coding method, and introduced a deletion algorithm into the genetic algorithm, which could provide emphasis for decision-makers [8]. Wang et al. proposed a path planning algorithm for arbitrary heading under the condition that flight safety is the first priority [9]. The dangerous degree of the grid is taken as the criterion of exploring node, and the practicability of the algorithm is proved by comparing it with the traditional algorithm. Wang et al. formed the NSGA-III algorithm based on the grid method path planning model combined with flow constraints and the redirection point's layout to avoid the potential conflict problem of multiaircraft redirection effectively [10]. Zhang et al. studied the optimal gait parameters combination of three gaits by using nondominated sorting genetic algorithm (NSGA-II) [11]. Orozco-Rosas et al. combined membrane computing with a genetic algorithm and the artificial potential field method to find the parameters to generate a feasible and safe path [12]. The MemPBPF algorithm proposed by Orozco-Rosas et al. contains dynamic membranes that include a pseudobacterial genetic algorithm for evolving the required parameters in the artificial potential field method [13]. The nondominated sorting genetic algorithm (NSGA-II) with elite retention strategy is applied to the research of path planning, a coding method suitable for path planning is proposed, and a deletion operator is introduced. The NSGA-III algorithm itself is an improvement of the genetic algorithm. On the basis of the second-generation algorithm, a new restriction condition based on traffic is added. The congestion degree is no longer used but a new population nondominated ranking is used to analyze individuals and solve path planning problems. It has high adaptability. Zheng et al. formed an improved A-star algorithm based on the idea of "tracking" to solve the problem that the optimal path of traditional A-star algorithm is "broken path." The algorithm uses the differential equation of the tracking model to solve the track and then "curved track" with a shorter distance than the initial "broken track" [14]. Bast et al. surveyed recent advances in algorithms for path planning in transportation networks [15]. Zhou et al. constructed an intelligent planning system for travel between cities based on the A-star algorithm [16]. Chen et al. proposed a weather path planning method based on the improved A-star algorithm; they reserved 5 search out-degree directions according to the starting point and the target point with a straight line and measure the angle $\alpha$ between the line and the true north direction [17].

van den Berg et al. presented a formal approach to reciprocal n-body collision avoidance, where multiple mobile robots need to avoid collisions with each other while moving in a common workspace [18]. Zhao used the simulated annealing algorithm intelligent algorithm as the guiding ideology of the path planning [19]. Singh and Willcox introduced an unmanned aerial vehicle path planning method using dynamic data to drive flight capability estimation [20]. Croce et al. used the VRP and the PCP as two sequential decision levels adopted by the same decision-maker [21]. Musolino et al. integrated the PCP and VRP levels through methodological and experimentation activities [22].

Although trajectory optimization method problems of UAVs are different from the aircraft path planning problem, there are similarities. Chen et al., based on mixed-integer linear programming, proposed an exact formulation to seek optimal flight paths for UAVs. Then, a clustering-based algorithm was presented to classify regions into clusters and to obtain feasible flight paths with the objective of minimizing the task completion time of UAVs [23]. Zhou et al. proposed an optimization method based on the Bessel curve's minimum and high-order displacement derivative trajectory [24]. Chen et al. introduced an exact formulation based on mixed-integer linear programming (MILP) to completely search the solution space with the models of UAVs and regions. Then, by improving the evaluation function and the node selection strategy, an improved $\mathrm{A} *$ algorithm is presented to produce an optimal flight path for UAVs. [25]. Ling et al. study the path planning problem of a UAV searching for multiple targets in the environment with irregular obstacles. They proposed an improved geometric algorithm (IGA) to optimize the length between different target points. Meanwhile, a search strategy based on the ant colony system (ACS) is proposed to find a search sequence with the shortest flying distance. The path length obtained by IGA-ACS is shorter, and the running time is very short [26]. Yang and Zhang examine the maneuver trajectory generation methods for UAVs based on energy optimization and design appropriate maneuver commands in the UAV flight envelope to achieve optimal energy performance [27].

The above analysis shows that the traditional path planning algorithms have problems such as too many search nodes, wide search directions, and long calculation time in aircraft flight path planning. A-star algorithm, D-star algorithm, and genetic algorithm are used in many documents, but the most prominent shortcoming of genetic algorithm is that it needs to encode and decode the problem properly, better convergence will lead to premature convergence, and the crossover rate and mutation rate of the genetic algorithm may have a great impact on the result. D-star algorithm and A-star algorithm can also achieve good results in the shortest path problem, but the time complexity of the D-star algorithm is much higher than the A-star algorithm. Therefore, we choose the heuristic A-star algorithm; A-star algorithm has good applicability, can be applied to path planning in various situations, and can ensure to find a feasible solution. By introducing the concept of a potential collision set, the velocity and acceleration set of the aircraft in the volcanic ash region are obtained, and the optimal velocity range of the aircraft is selected from the finite element models. The flight trajectory is calculated by adding displacement increment, and the $F(n)$ value is refreshed continuously to get the final path planning result. 
Compared with other path planning algorithms, the A-star algorithm has the following outstanding advantages:

(1) A-star algorithm is a heuristic algorithm optimized by the Dijkstra algorithm. Compared with other kinds of algorithms, the solution of the A-star algorithm is accurate and efficient, and the grid path is evaluated by the evaluation function to select the optimal path, without falling into the defect of local optimization. Compared with other heuristic algorithms, the A-star algorithm has the least number of nodes and the least amount of calculation.

(2) A-star algorithm mainly relies on heuristic functions for pathfinding. In different rerouting scenarios, different evaluation functions can be selected to improve the search strategy of the A-star algorithm.

(3) A-star algorithm is also more adaptable to the environment; it can be improved by combining the advantages of other algorithms to achieve the purpose of finding the best path more effectively.

The A-star algorithm also has certain defects. Due to the problem of the A-star algorithm search strategy, when it finds the best iteration point, it usually expands from the parent node to every node around it, which causes a large number of unnecessary node calculations, When the accuracy of the local map is higher, the map grid division is smaller, which increases the amount of calculation. Because the grid method is a grid-type path connection, sometimes it will cause the transition and turning, which is not conducive to the actual aircraft movement.

The ORCA algorithm can meet the requirements of realtime motion planning and obstacle avoidance in dynamic environments [28]. The main idea is to select the speed outside the speed barrier region, and the speed gives a linear speed selection region (ORCA region) with the current two agents [29].

The limitations of the ORCA algorithm are mainly in the following three points:

(1) The decision time of the ORCA algorithm increases with the increase of the number of motions, and it is unable to deal with the problem of large-scale agents well in a short time

(2) ORCA algorithm does not always find a reasonable speed linear selection region, and sometimes, some approximate regions are needed to replace it, which will lead to some obstacle avoidance selections of the ORCA algorithm which are not optimal

(3) ORCA algorithm does not take into account the kinematic constraints of the aircraft very well [29]

A-star algorithm is exactly complementary to the ORCA algorithm:

(1) ORCA algorithm is a local path planning method, which aims to make individuals avoid other individual goals and obstacles that are close to themselves. ORCA algorithm can only perceive the situation close to itself; without global environment information, it cannot find the shortest path between the starting point and the target point, which is exactly the problem solved by the A-star algorithm.

(2) A-star algorithm is a global pathfinding algorithm, which can find the shortest path from the starting point to the target point. The global information of the algorithm contains the obstacle information of the whole environment. But A-star algorithm cannot perceive the specific surrounding conditions of all individuals, so the A-star algorithm cannot handle the collision problem, which happens to be the problem solved by the ORCA algorithm.

\section{Path Planning}

The A-star algorithm is one of the most commonly used methods in static path planning. In order to solve the problem of the impact of volcanic ash on flight safety, the velocity vector model of the volcanic ash region is established, and the optimal speed range is found under the conditions of monitoring the diffusion and transfer of volcanic ash. Then, using the vector idea of the ORCA algorithm to improve the A-star algorithm can make the aircraft bypass the threat area perfectly and ensure flight safety.

For easier reading, the variables and technical terms used in this paper are defined and explained in Table 1.

3.1. Volcanic Ash Model. Let us assume that each ash field is a circle. In order to ensure flight safety, as far as possible to cover the center of the condensation core volcanic ash region, the area covered by volcanic ash is a three-dimensional space, which can be divided into two-dimensional planes according to different flight levels. Assuming that all volcanic ash areas are set as $B$, each volcanic ash area $B_{i}$ has a current position $P_{B i}$ (position of volcanic ash condensation nuclei), current velocity $V_{B i}$, and circular radius (radius of volcanic ash clouds) $R_{B i}$. The ash field covers an area that can be detected by satellite and can be fed back to aircraft that have encountered the ash. These are the parameters that can be observed by the aircraft. In addition, it is assumed that each volcanic ash area will not be affected by other volcanic ash or other factors along the course of the current speed direction; that is, all volcanic ash areas are independent and unrelated. The airplane has a maximum speed $V_{A}$ max and the desired speed $V_{A}$ exp, which are limited by the performance of the airplane. The direction of $V_{A} \exp$ points to the target airport of the airplane, and the ideal length of $V_{A}$ exp is equal to $V_{A} \max$. The speed vector synthesis diagram is shown in Figure 1.

In Figure 1, $A$ is flying forward, and $V_{A}$ represents the speed vector of the airplane at the current position. To prevent the aircraft from contacting the volcanic ash area and achieve the purpose of path planning in a dynamic environment, the concept of a potential collision set is introduced. The elements of this set include all volcanic ash areas that may overlap with the aircraft in the future $T$ time. 
TABLE 1: Abbreviation table.

\begin{tabular}{|c|c|}
\hline Symbol & Description \\
\hline$\overline{F(n)}$ & The total cost from the global starting point to the global target point \\
\hline$g(n)$ & The cost of the shortest path from the global starting point to the current position \\
\hline$h(n)$ & The estimated cost of the shortest path from the current position to the global target point \\
\hline$P_{B}$ & Position of volcanic ash condensation nuclei \\
\hline$V_{B}$ & The speed of volcanic ash \\
\hline$R_{B}$ & Radius of volcanic ash clouds \\
\hline$B$ & Volcanic ash area \\
\hline$V_{A}$ & The speed of an airplane \\
\hline$N$ & The exploration node in the running space of the algorithm \\
\hline ORCA & Optimal reciprocal collision avoidance \\
\hline$P(n)$ & The possibility of node $x$ \\
\hline$W_{i}$ & The node to be explored in the next step \\
\hline$W_{j}$ & The node of the final destination of the aircraft \\
\hline$P$ & The global starting point \\
\hline Q & The global target point \\
\hline$M$ & The linear distance between the current position and the global target point \\
\hline$s$ & Current starting point \\
\hline$D$ & Path length \\
\hline$\Theta$ & Change of angle \\
\hline$H$ & Distance off course \\
\hline$T$ & Run time \\
\hline$r_{i}$ & The turning point \\
\hline$U_{\text {rep }}(q)$ & Repulsive potential field \\
\hline$k_{\text {rep }}$ & Repulsion scale factor \\
\hline$\rho\left(q, q_{0}\right)$ & Distance from obstacle to aircraft \\
\hline$\rho_{0}$ & The range of influence of the obstacle \\
\hline
\end{tabular}

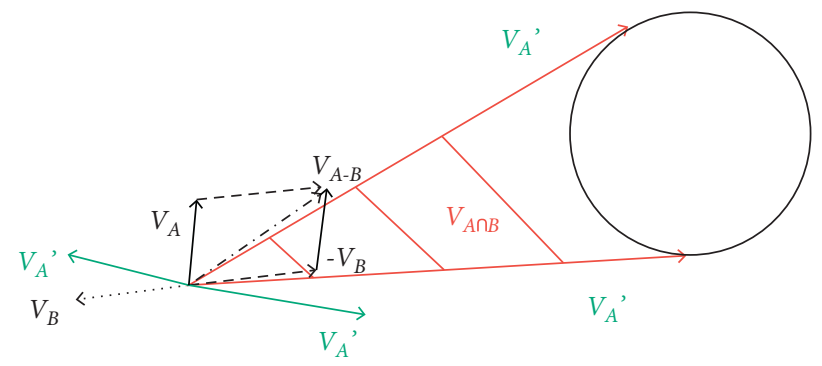

Figure 1: Speed vector synthesis diagram.

The ash is only moved by the influence of the wind, and the information of wind speed and wind direction is very easy to get. So $V_{B}$ represents the speed vector of the volcanic ash area. For airplanes, it is $V_{A} \exp -V_{B} \exp . V_{A \cap B}$ represents the speed at which $A$ and $B$ will overlap. It is the potential collision set we just said. $V_{A}{ }^{\prime}$ represents the speed at which the two do not overlap, which is the speed at which it is perfectly safe. The aircraft must consider the speed of all volcanic ash areas and try to avoid the risk of collision with them. Of course, this speed needs to be within the speed range that meets the performance conditions.

Another point to note is that for airplanes, the direction of flight speed cannot be omnidirectional, and each airplane can only have an optional half-plane speed. At the same time, each aircraft has its own maximum acceleration and deceleration. Although there are a limited number of combinations in the two-dimensional set of velocities and acceleration $\left(V_{x}, V_{y}\right)$, how to choose the best speed from the intersection of these optional multiple planes, especially when the linear division may not find the optimal solution under such complex conditions, we need to choose the "as safe as possible" speed. Because the acceleration and deceleration of the aircraft have a relatively small effect on the speed in a short time, changing the speed direction of the aircraft seems to be a more reliable way.

The ORCA algorithm is a local navigation method, which is based on the perception of the surrounding information and does not overlap the obstacle area. However, the ORCA algorithm can not find the shortest path from the beginning to the end of the aircraft. This is exactly the kind of problem that the A-star algorithm can solve. In addition, the ORCA algorithm can improve the efficiency of the A-star algorithm to explore nodes. The ORCA algorithm can ensure the aircraft always moving toward the target and avoid the obstacle area in the process. For the area of volcanic ash, airplane $A$ can be regarded as a moving particle. As long as it is ensured that the speed direction of $A$ does not overlap with the volcanic ash area $B$, there will be no collision within a 
certain period of time. $V_{A-B}$ represents the combined vector of $V_{A}$ and $-V_{B}$ speed. When $V_{A-B} \in V_{A \cap B}, A$ and $B$ must have overlapping areas. When $V_{A-B} \in \notin V_{A \cap B}$, there will be no intersection between $A$ and $B$.

An aircraft in flight state will have a minimum speed to maintain the flight state and a maximum speed limited by the model. All speeds in this range are represented by sets.

$$
V_{S}=\left\{V \in\left[V_{\min }, V_{\max }\right]\right\} \text {. }
$$

The critical velocity vector is the velocity when there is only a tangent point between the velocity and the obstacle area, and the critical velocity vector must exist in the velocity set $V_{S}$.

When calculating the aircraft trajectory, we first consider two adjacent moments and regard the motion trajectory between two adjacent points as a straight line, which is equivalent to moving $V t * \Delta t$ in the forward direction of the aircraft. We just project the distance to the $x$-axis and $y$-axis of the coordinate system to get the displacement of the aircraft in the coordinate system at time $t+1$ relative to time $t$.

$$
\begin{aligned}
& \Delta x=v \cdot \Delta t \cdot \cos \left(\theta_{t}\right), \\
& \Delta y=v \cdot \Delta t \cdot \sin \left(\theta_{t}\right) .
\end{aligned}
$$

By analogy, the cumulative sum of the displacement increments in a period of time can calculate the distance traveled by aircraft during this period.

$$
\begin{aligned}
& x=x_{0}+v \cdot \Delta t \cdot \cos \left(\theta_{t}\right), \\
& y=y_{0}+v \cdot \Delta t \cdot \sin \left(\theta_{t}\right), \\
& \theta_{t}=\theta_{t 0}+w \cdot \Delta t,
\end{aligned}
$$

where $x_{0}$ is the abscissa before the displacement of the aircraft, $x$ is the abscissa after the displacement of the aircraft, $y_{0}$ is the ordinate before the displacement of the aircraft, and $y$ is the ordinate after the displacement of the aircraft. The $\theta_{t 0}$ is the vertical coordinate before the displacement of the aircraft and $\theta_{t}$ is the vertical coordinate after the displacement of the aircraft.

The perception and reaction cycle of the aircraft is shown in Figure 2.

The evaluation function is shown as

$$
\gamma=\alpha \cdot \operatorname{dist}(V, \omega)+\beta \cdot \operatorname{velocity}(V, \omega) .
$$

$\operatorname{Dist}(V, w)$ represents the distance between the aircraft's current position and the nearest volcanic ash area, and velocity $(V, \omega)$ represents the aircraft's speed and vector direction at the current position.

Finally, we obtained

$$
F(n)=g(n)+\gamma \cdot h\left(n_{x}, n_{y}\right)
$$

3.2. Improved A-Star Algorithm. A-star algorithm formula can be expressed as follows:

$$
F(n)=g(n)+h(n),
$$

where $F(n)$ represents the total cost from the global starting point to the global target point, $g(n)$ is the cost of the shortest path from the global starting point to the current position, and $h(n)$ is the estimated cost of the shortest path from the current position to the global target point. $n$ is the exploration node in the running space of the algorithm. The exact coordinates are $n\left(n_{x}, n_{y}\right)$. The values of $x$ and $y$ are the same as in the above section.

The searching process of the A-star algorithm is shown in Figure 3.

When $h(n)=0$, the A-star algorithm degenerates to the Dijkstra algorithm. While $h(n)$ is much larger than $g(n)$, the A-star algorithm is transformed into the DFS algorithm. If the actual $h(n)$ differs from the ideal $h(n)$, the operating efficiency of the A-star algorithm is also lower, so it is necessary to give a reasonable direction to the a priori of $h(n)$.

$$
g(n)=P(n) *(g(n)-1)+1,
$$

where $g(n)$ represents the actual cost after the update and $g^{\prime}(n)$ represents the actual cost before the update. $P(n)$ represents the possibility of node $x$.

If point $n$ is an area covered by volcanic ash, we take $P(n)=0$. At this time, the exploration node of $g(n)$ will increase by 1 and continue to explore. If point $n$ is not covered by volcanic ash, the aircraft can pass, take $P(n)=1$, and update the value of $g(n)$.

Assuming that there are $i$ volcanic ash-covered areas located near the starting and ending points of the path, due to the high-altitude wind, the nature of the volcanic ash particles, and other factors, the size and degree of influence of the volcanic ash covered areas are different. $n$ represents the current position of the aircraft, and $j$ represents the position of the global target point. Propose $h(n)$ as follows:

$$
h(n)=h^{\prime}\left(n, W_{i}\right)+\operatorname{distance}\left(W_{i}, W_{j}\right),
$$

where $h(n)$ represents the actual cost after the update and $h^{\prime}(n)$ represents the actual cost before the update. $n$ is the node of the current position of the aircraft, $W_{i}$ is the node to be explored in the next step, and $W_{j}$ is the node of the final destination of the aircraft. $h^{\prime}\left(n, W_{i}\right)$ represents the estimated cost of moving from the current location $n$ to the next node $W_{i}$, and distance $\left(W_{i}, W_{j}\right)$ represents the distance from the next node $W_{i}$ to the final target point $W_{j}$. The optimization of $F(n)$ is achieved by continuously reducing the estimated cost of $h(n)$ and transforming it into increasing the actual cost of $g(n)$. The choice of $W_{i}$ is explained by algorithm steps.

Different from other algorithms that require centralized control, the ORCA algorithm is a more decentralized and more practical algorithm because of its lower hardware requirements and simpler calculations. Use the speed vector idea of the ORCA algorithm to improve the $h(n)$ function of the A-star algorithm. Suppose that an airplane is flying in a space environment with fixed information (start and end points are known, static obstacles are known, and dynamic obstacles are unknown). Volcanic ash will spread and transfer with wind speed and direction due to its different 


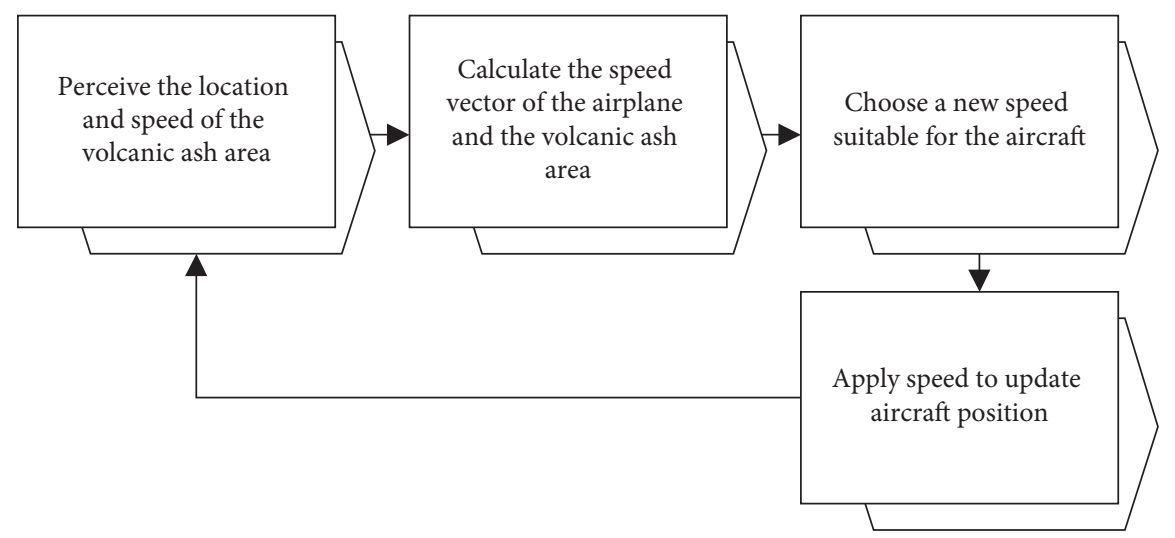

FIGURE 2: The cyclic process of the aircraft sensing the volcanic ash area and processing it.

\begin{tabular}{|ll|ll|ll|}
\hline$F\left(n_{4}\right)$ & & $F\left(n_{3}\right)$ & & $F\left(n_{2}\right)$ & \\
$G\left(n_{4}\right)$ & $H\left(n_{4}\right)$ & $G\left(n_{3}\right)$ & $H\left(n_{3}\right)$ & $G\left(n_{2}\right)$ & $H\left(n_{2}\right)$ \\
\hline$F\left(n_{5}\right)$ & & & $F\left(n_{1}\right)$ & \\
$G\left(n_{5}\right)$ & $H\left(n_{5}\right)$ & & Current point & & \\
\hline$F\left(n_{6}\right)$ & & $F\left(n_{7}\right)$ & $G\left(n_{1}\right)$ & $H\left(n_{1}\right)$ \\
\hline$G\left(n_{6}\right)$ & $H\left(n_{6}\right)$ & $G\left(n_{7}\right)$ & $H\left(n_{7}\right)$ & $G\left(n_{8}\right)$ & $H\left(n_{8}\right)$ \\
\hline
\end{tabular}

FIGURE 3: Searching process by A-star algorithm.

mass, resulting in different obstacle areas. This process is slow. Compared with the flight process of a single aircraft, the volcanic ash area can also be regarded as a static obstacle.

The specific steps of the improved A-star algorithm are as follows:

(1) Know the global starting point $P$ and the global target point $Q$, initialization parameters.

(2) Calculate the linear distance $M$ between the current position and the global target point, and use Euclidean distance calculation.

(3) $F(n)$ represents the total cost from the global starting point to the global target point, $g(n)$ is the cost of the shortest path from the global starting point to the current position, and $h(n)$ is the estimated cost of the shortest path from the current position to the global target point.

(4) Assuming an ideal path $F(n) \min =g(n)$ from the current start point to the global end point, the speed direction of the aircraft at this time is from the global departure point to the global target point.

(5) If there is a single volcanic ash coverage area, move the line connecting the global starting point and the global target point to both sides along the normal direction until the point on the straight line is exactly the line connecting the starting and ending points and there is only one tangent point to the volcanic ash clouds area. Get two tangent points between the line and the volcanic ash-covered area, as shown in Figure 4.

(6) If there are multiple volcanic ash covered areas at the same time, calculate the distance $M\left(s P_{B}\right)$ between the current starting point and the center of each obstacle, and move the line along the normal direction until the volcanic ash covered area that is closest to the current starting point and has an intersection with the original line. There are exactly two tangent points or stops when the preset boundary is reached. The principle is the same as step 5.

(7) Compare the distance from the current position to the two tangent points and select the relatively better point by calculating the evaluation function.

(8) Set the tangent point 1 to $n_{1}$, and make the connection from the current starting point to the tangent point $n_{1}$ (the direction of the connecting line in dynamic path planning is $V_{A-B}$, as shown in Figure 5).

If the line does not intersect with other obstacle circles, calculate $F\left(n_{1}\right)$ at this time. If the line has an intersection point $n_{2}$ with other obstacle circles, change it to the line from the current starting point to the intersection point $n_{2}$ and recalculate until the recalculated line is only tangent to the obstacle circle.

(9) Regarding $n_{i}$ as the current starting point, repeat Steps $4,5,6$, and 7 . If $M>0$, continue to 3 ; if $M$ is equal to 0 , stop and go to step 10 .

Calculate the final $F(n)$, which is the actual function from the global starting point to the global target point.

3.3. Algorithm Implementation. The implementation flowchart of the improved A-star algorithm is shown in Figure 6:

Step 1: the algorithm starts. If the path is just not covered by the volcanic ash area, the spread and 


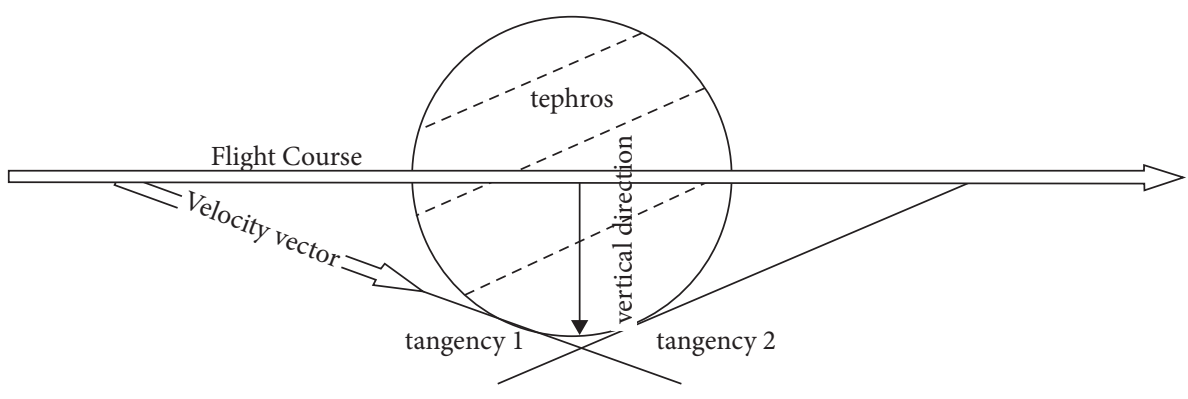

Figure 4: Cut point of a single volcanic ash area.

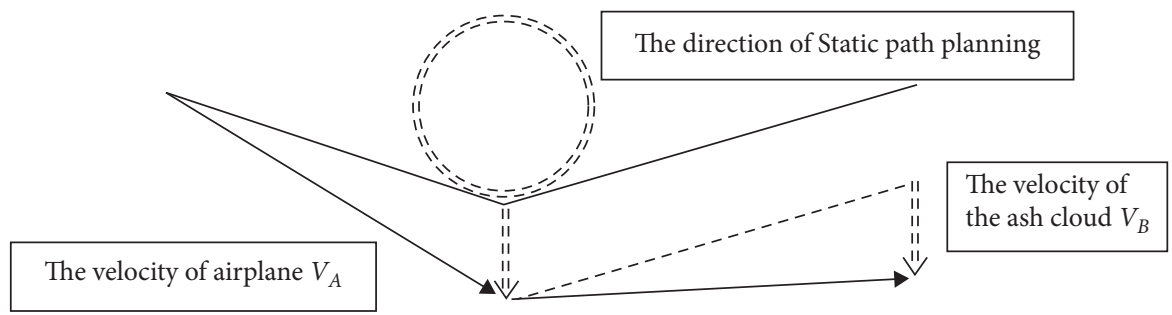

FIgUre 5: Path planning when the ash cloud moves.

transfer of volcanic ash caused by other factors will not affect the path. Calculate the ideal condition $F(n)$.

Step 2: at this time, $h(n)=0, F(n)_{\min }=g(n)$. If the volcanic ash area will affect the path, move the line connecting the global starting point and the global target point along the normal direction until there is only one intersection with the volcanic ash area.

Step 3: use the intersection as the intermediate node of the path planning to determine the velocity vector, and determine the displacement increment through the velocity vector to obtain the updated $F(n)$.

Step 4: to plan the path from the global starting point to the intermediate node and the path from the intermediate node to the global target point, continuously update $F(n)$ through the previous steps of the cycle to complete the path planning.

The pseudocode of the implementation program is shown in Figure 7.

The return value is directly replaced with the original value to achieve the purpose of updating $F(n)$.

According to the improved A-star algorithm, for each volcanic ash cloud, only consider flying around two boundaries, so that we just need to compare the two paths. The searching process by improved A-star algorithm is shown in Figure 8.

3.4. The Objective Function. Considering factors such as air traffic control procedures and flight performance of aircraft during rerouting flight, we select four representative objective functions, which are path length $D$, change of angle $\Theta$ (the smaller the $\Theta$, the easier it is for the pilot to operate), distance off course $H$ (the smaller the $H$, the higher the airspace utilization), and run-time $T$, respectively. The objective functions of multiobjective programming are shown as

$$
\begin{aligned}
& \operatorname{Min} D=d\left(p, r_{1}\right)+d\left(r_{1}, r_{2}\right)+\cdots d\left(r_{n-1}, r_{n}\right)+d\left(r_{n}, Q\right), \\
& \operatorname{Min} \Theta=\theta\left(p, r_{1}\right)+\theta\left(r_{1}, r_{2}\right)+\cdots \theta\left(r_{n-1}, r_{n}\right)+\theta\left(r_{n}, Q\right),
\end{aligned}
$$

$\operatorname{Min} H$,

$\operatorname{MinT}$.

The result of path planning is $P-r_{1}-r_{2} \ldots r_{n}-Q, P$ is the starting point, $Q$ is the end point, and $r_{i},(i=1,2 \ldots n)$ is the turning point.

\section{Simulation and Results Analysis}

The ash clouds from the 2010 Icelandic volcano affected the skies over Europe, prompting all European airlines to suspend flights for five days, arranging test flights as soon as conditions improved slightly, and gradually resume more flights around the volcanic ash zone normal flight.

The simulation experiment environment is shown in Figure 9.

It can simulate the eruption process by volcanic crater location, crater size, volcanic eruption intensity, wind speed and direction during a volcanic eruption, and other factors. The direct flight path selects Madrid to Cairo and Algiers to Rome as simulation cases and selects the volcanic ash clouds at the cruise level of the path for horizontal analysis.

The simulation tool used in this paper is KWare Erupt3, which is an interface-type software for simulating volcanic eruptions. The procedures of the experiment are as follows:

Step 1: collect the data of the route and volcano, mark the route and the volcanic ash area on the map, and build the experimental scene. 


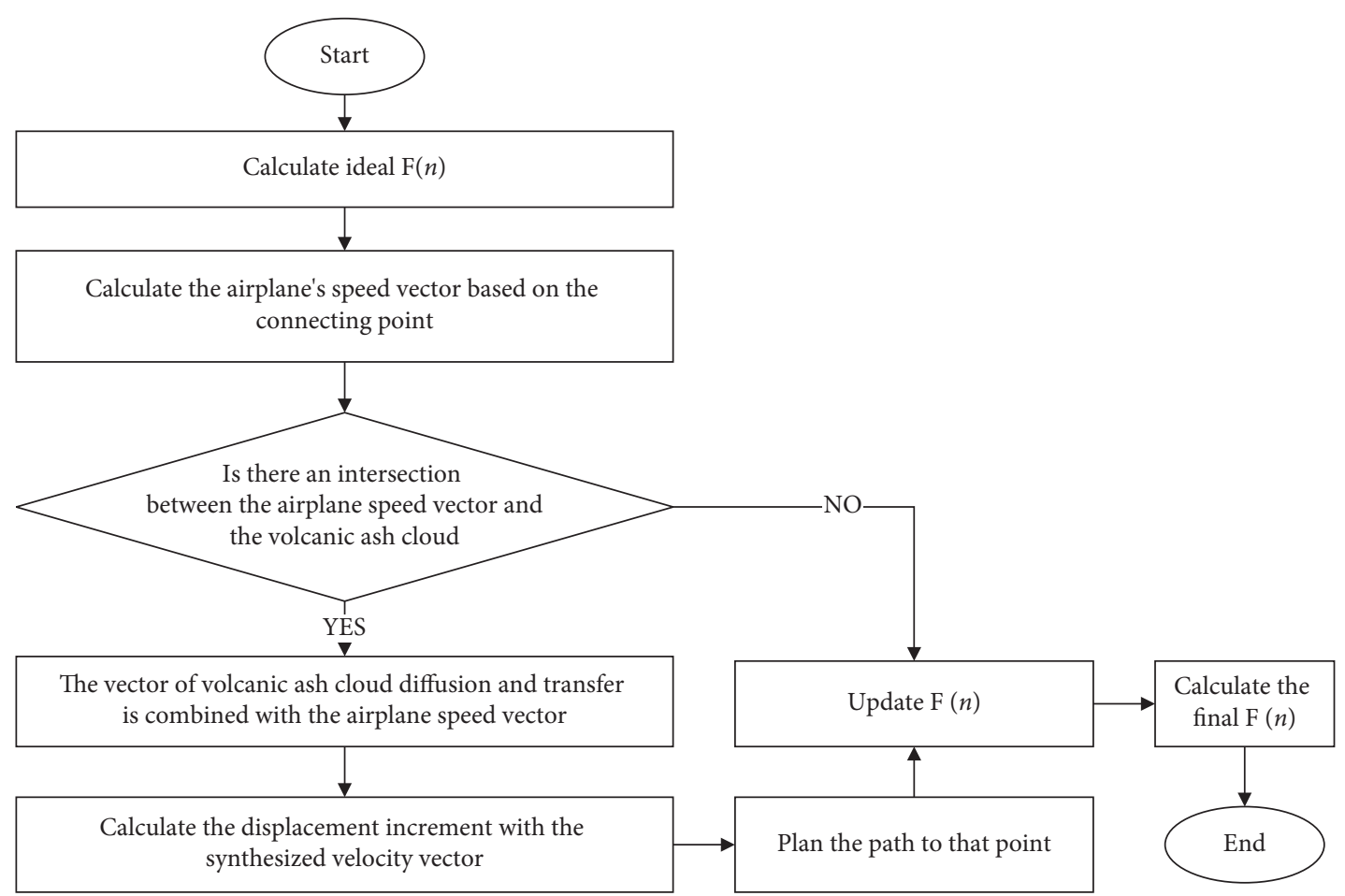

FigURE 6: Technical path of the improved A-star algorithm for velocity vector.

$$
\begin{array}{ll}
\hline \text { Input: } & P=\left(B_{P}, L_{P}\right), Q=\left(B_{Q}, L_{Q}\right), V_{A}=\left(V_{A x}, V_{A y}\right) \\
& \text { data set } B=\left\{\left(B_{1}, L_{1}, R_{1}\right),\left(B_{2}, L_{2}, R_{2}\right), \ldots,\left(B_{i}, L_{i}, R_{i}\right)\right\}
\end{array}
$$

Output: node of path

For $n=$ new node do

if $\mathrm{n}$ in $B$, then

$V_{A}=0$

return $n$

end

else

$V_{A}=$ new $V_{A}$

put back $V_{A}$

end

end

Figure 7: Program pseudocode.

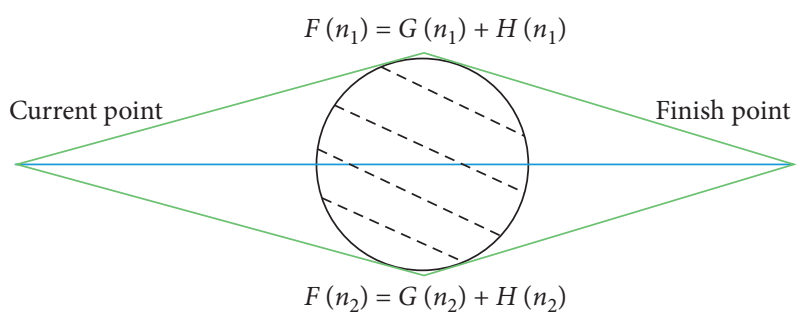

FIGURE 8: Searching process by the improved A-star algorithm. 


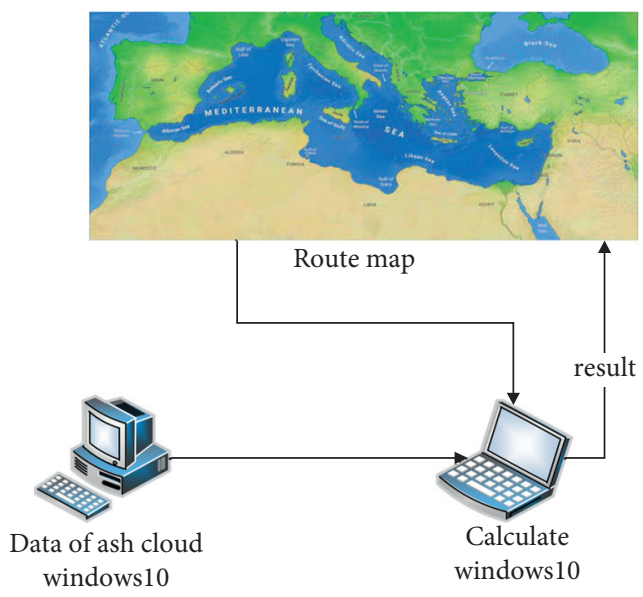

FIGURE 9: Simulation experiment environment.

Step 2: use the above algorithm for path planning and consider volcanic ash clouds do not move.

Step 3: compare and analyze the results.

Step 4: considering the movement of volcanic ash, adjust the results of the static path planning.

Madrid is the capital of Spain and it is located in central Spain with the latitude and longitude coordinates being $\left(40^{\circ} \mathrm{N}, 3^{\circ} \mathrm{W}\right)$. Cairo is the capital of Egypt and it is located in the northeast of Egypt with the latitude and longitude coordinates being $\left(30^{\circ} \mathrm{N}, 31^{\circ} \mathrm{E}\right)$. The latitude and longitude coordinates of the center of the volcanic ash area and the circular radius of the volcanic clouds are shown in Table 2.

The known spread and transfer of volcanic ash clouds after the volcanic eruption are shown in Figure 7 . The circle represents the area covered by volcanic ash, and the larger the radius of the circle, the larger the range of volcanic ash in the area.

As shown in Figure 10, track 1 and track 2, respectively, represent the simulation results of selecting the velocity vector interval at the boundary of No.2 and No.4 volcanic ash areas.

The tangent point is obtained after translation along the normal direction from the connecting line of the starting and ending points, the velocity vector interval is determined by the tangent point, and the final track is obtained by substituting it into the algorithm. Track 3 represents the track after selecting a more optimized track from track 1 and track 2 and smoothing.

As shown in Figure 11, track 1 and track 2 represent the simulation results of selecting the velocity vector interval at the boundary of the No.1 and No.4 volcanic ash areas, respectively.

It can be clearly seen from Figure 11 that the line between the first tangent point and the starting point obtained after translation along the normal direction will intersect the No.2 and No.5 volcanic ash areas, so it is necessary to continue to translate the connection along the normal direction. Track 3 represents the track after selecting a more optimized track from track 1 and track 2 and smoothing. The distances of the three trajectories from Madrid to Cairo are shown in Table 3.
TABLE 2: Latitude and longitude coordinates and radius of the volcanic ash center.

\begin{tabular}{lccc}
\hline Volcanic ash area & Longitude & Latitude & Radius $(\mathrm{km})$ \\
\hline 1 & $2.9^{\circ} \mathrm{E}$ & $36.9^{\circ} \mathrm{N}$ & 110 \\
2 & $14.5^{\circ} \mathrm{E}$ & $36.7^{\circ} \mathrm{N}$ & 200 \\
3 & $28.1^{\circ} \mathrm{E}$ & $35.4^{\circ} \mathrm{N}$ & 160 \\
4 & $22.8^{\circ} \mathrm{E}$ & $32.1^{\circ} \mathrm{N}$ & 110 \\
5 & $12.9^{\circ} \mathrm{E}$ & $32.0^{\circ} \mathrm{N}$ & 200 \\
\hline
\end{tabular}

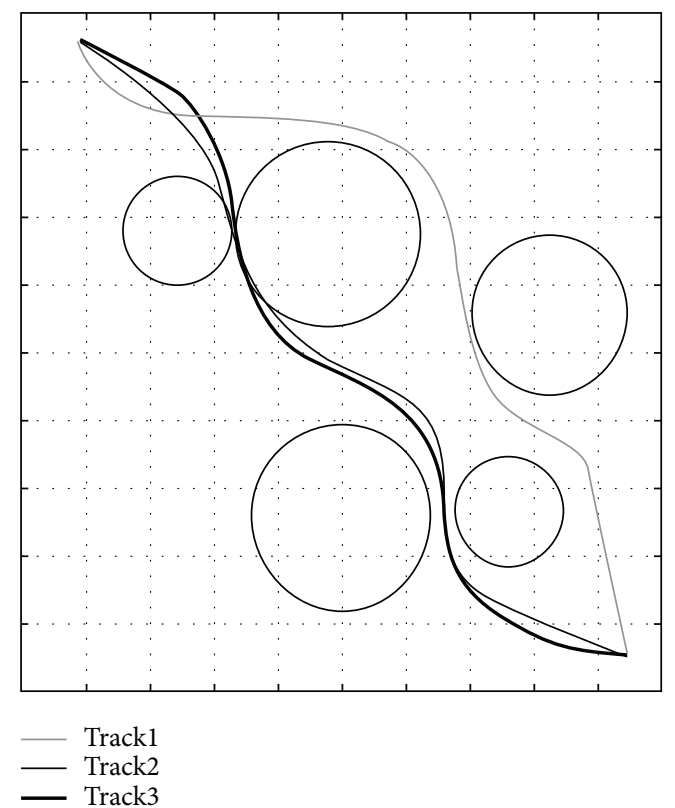

Figure 10: Path 1 covered by volcanic ash.

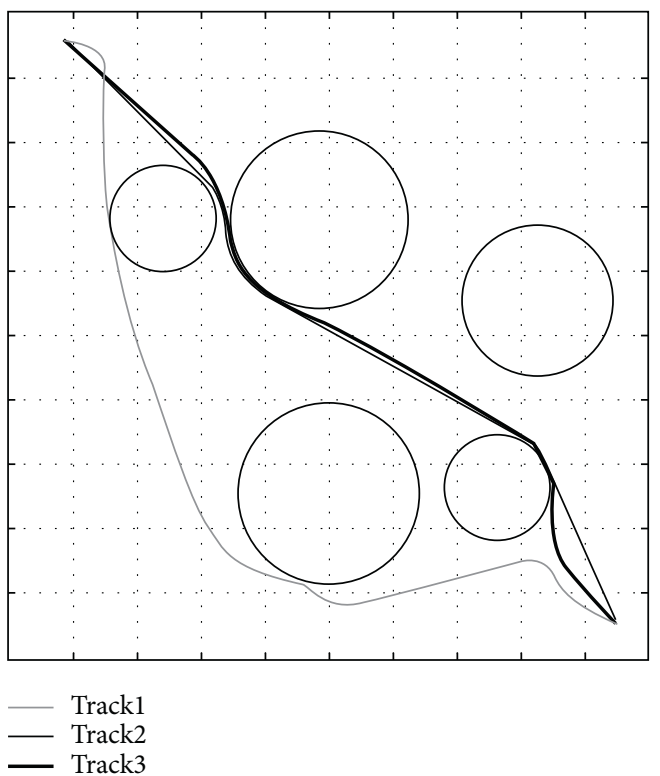

Figure 11: Path 2 covered by volcanic ash.

The length of the three trajectories in the simulation result of Figure 10 is represented by the trajectory distance one, and the length of the three trajectories in the simulation 
result of Figure 11 is represented by the trajectory distance two.

In addition, the same analysis is carried out on the path from Algiers (the capital of Algeria) to Rome (the capital of Italy). Algiers is located in the northern part of Algeria with the latitude and longitude coordinates of $36^{\circ} \mathrm{N}, 3^{\circ} \mathrm{E}$. Rome is located in central and western Italy with the latitude and longitude coordinates of $39^{\circ} \mathrm{N}, 12^{\circ} \mathrm{E}$. The longitude and latitude coordinates of the center of the volcanic ash area and the volcanic clouds circle radius are shown in Table 4.

The track results are shown in Figure 12.

In Figure 12, track 1 and track 2 represent the simulation results of selecting the velocity vector interval at the boundary of the volcanic ash area No.2 and No.5, respectively. The tangent point is obtained after translation along the normal direction from the connecting line of the starting and ending points, the velocity vector interval is determined by the tangent point, and the final track is obtained by substituting it into the algorithm. Track 3 represents the track after selecting a more optimized track from track 1 and track 2 and smoothing. The three track length results are shown in Table 5.

From the analysis of the above simulation results, the following conclusions can be drawn:

(1) From the case of Madrid to Cairo, it can be seen that the tangent point between the parallel movement of the algorithm and the volcanic ash area will affect the final result of the track, but the track 2 distance is less than the track 1 distance in both cases. It shows that the smaller the translation distance along the normal direction, the smaller the additional distance that the track exceeds the path, which is more in line with the actual operating conditions.

(2) Two boundary vectors will appear in the collection of velocity vectors generated in the same volcanic ash area. The smaller the angle between the direction of the velocity vector and the unit vector connecting the starting and ending points, the closer the resulting final track distance will be to the course distance. The larger the unit vector angle between the starting and ending points, the farther the final track distance will be.

(3) From the case of Algiers to Rome, it can be seen that the final track results conform to the law, but the first half of track 2 fails to strictly follow the algorithm. The reason is that the starting and ending points are relatively close, and the volcanic ash area is where the starting and ending points pass through. More often, the locally optimal solution is easy to appear when calculating the velocity vector, so that the result of track 2 does not satisfy the global optimal solution, but it satisfies the global feasible solution. Relative to track 1 , track 2 is still a more optimized track result.

In order to reflect the superiority of the algorithm and the fairness of the comparison, different path planning algorithms are used to plan the path from Madrid to Cairo and the path from Algiers to Rome. The traditional A-star
TAble 3: Three trajectory distances from Madrid to Cairo.

\begin{tabular}{lcc}
\hline Track & Track distance one $(\mathrm{km})$ & Track distance two $(\mathrm{km})$ \\
\hline 1 & 3660 & 3898 \\
2 & 3579 & 3576 \\
3 & 3525 & 3474 \\
\hline
\end{tabular}

TABLE 4: Latitude and longitude coordinates and radius of the volcanic ash canter.

\begin{tabular}{lccc}
\hline Volcanic ash area & Longitude & Latitude & Radius $(\mathrm{km})$ \\
\hline 1 & $3.8^{\circ} \mathrm{E}$ & $36.5^{\circ} \mathrm{N}$ & 50 \\
2 & $5.8^{\circ} \mathrm{E}$ & $36.8^{\circ} \mathrm{N}$ & 80 \\
3 & $7.5^{\circ} \mathrm{E}$ & $38.2^{\circ} \mathrm{N}$ & 120 \\
4 & $7.9^{\circ} \mathrm{E}$ & $35.0^{\circ} \mathrm{N}$ & 110 \\
5 & $10.3^{\circ} \mathrm{E}$ & $37.8^{\circ} \mathrm{N}$ & 90 \\
\hline
\end{tabular}

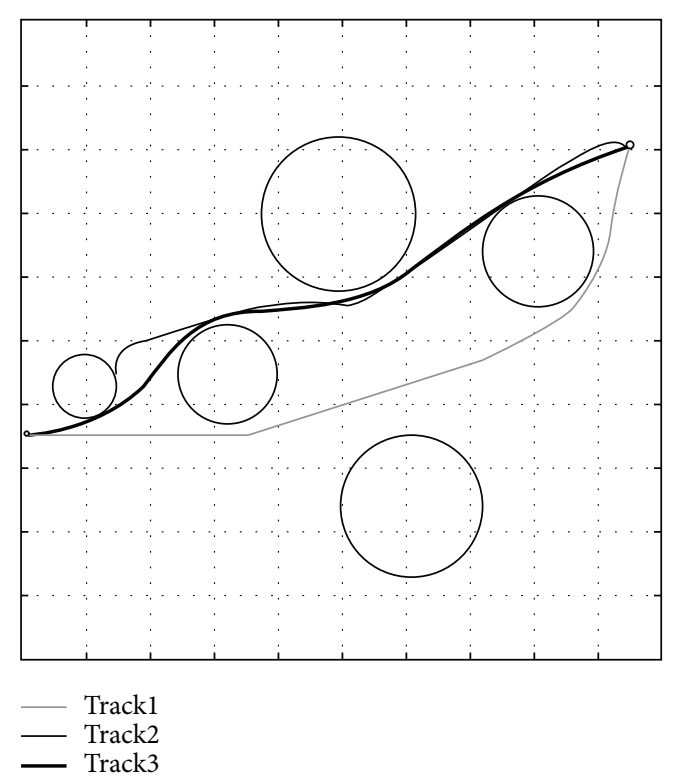

Figure 12: Path 3 covered by volcanic ash.

TABle 5: Three trajectory distances from Algiers to Rome.

\begin{tabular}{lc}
\hline Track & Track distance $(\mathrm{km})$ \\
\hline 1 & 1120 \\
2 & 1148 \\
3 & 1095 \\
\hline
\end{tabular}

algorithm, artificial potential field method, and genetic algorithm are used for simulation many times, and the running environment of these algorithms is identical. The searching process of the improved A-star algorithm is shown in Figures 13-15.

The searching process of the traditional A-star algorithm and genetic algorithm is shown in Figures 16-18. The Arabic numerals in the figure indicate the search order.

The results of path planning from Madrid to Cairo are shown in Table 6 and Figure 19.

The results of path planning from Algiers to Rome are shown in Table 7 and Figure 20. 


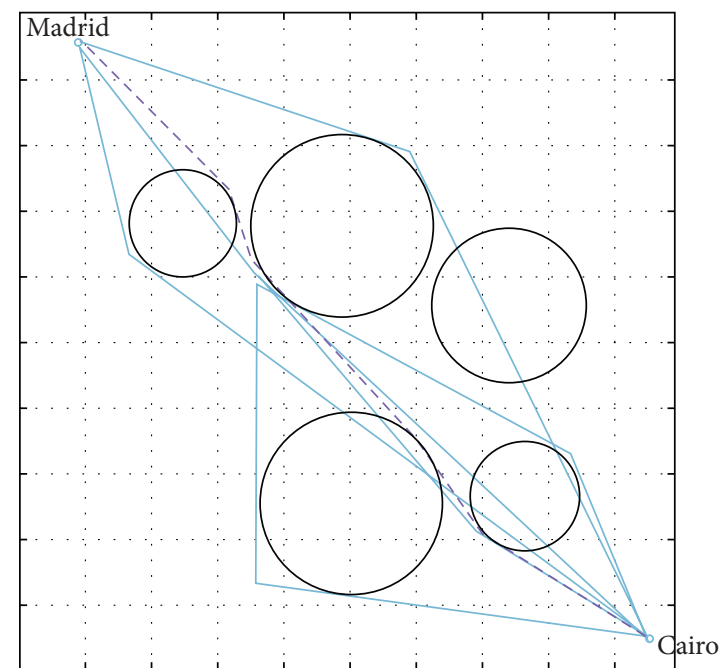

- - - Shortest path

Other searching path

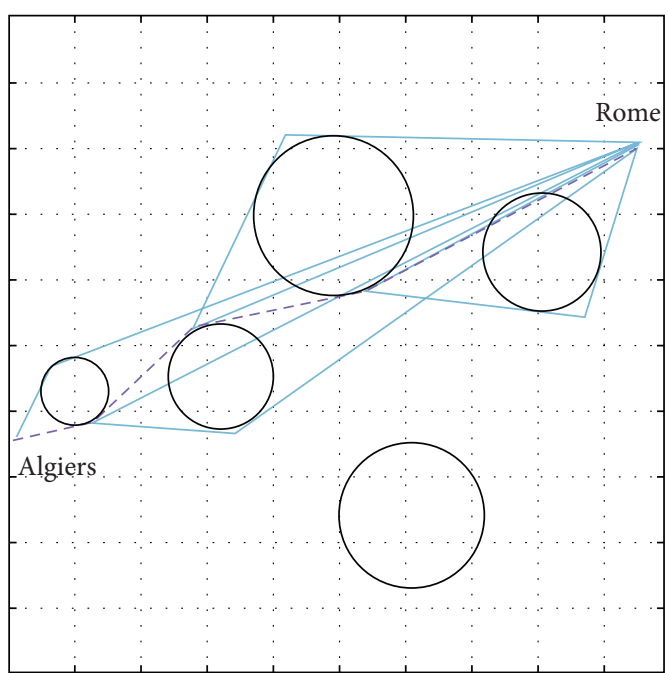

- - - Shortest path

Other searching path

FIGURE 13: Searching process of the improved A-star algorithm.

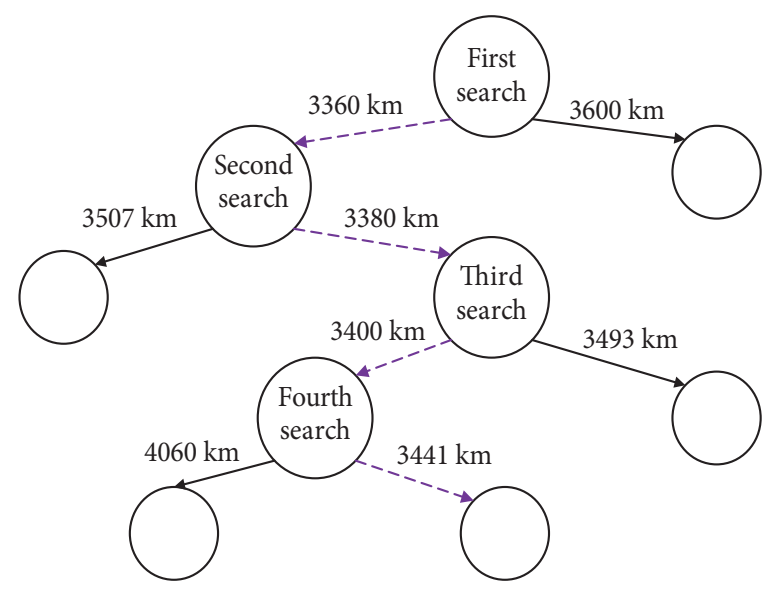

Shortest path $\quad--\rightarrow$
Other searching path $\longrightarrow$

Figure 14: Search path $F(n)$ from Madrid to Cairo.

The two scenarios are different. The length of the path from Madrid to Cairo is approximately three times that from Algiers to Rome, so in the scenario of Madrid to Cairo, the off-course distance is longer. In addition, the gap between ash clouds No.1 and No.2 is small, resulting in a generally small distance between aircraft and the ash clouds.

It can be seen that the path planning results of the improved A-star algorithm are better than other several algorithms; in descending order, they are the improved A-star algorithm, genetic algorithm, traditional A-star algorithm, and artificial potential field method.

The repulsive potential field function is shown as

$$
U_{\text {rep }}(q)=\frac{1}{2} k_{\text {rep }}\left[\frac{1}{\rho\left(q, q_{0}\right)}-\frac{1}{\rho_{0}}\right]^{2} 0 \leq \rho\left(q, q_{0}\right) \leq \rho_{0} .
$$

The reason for the bad effect of the artificial potential field method is that the volcanic ash has a large area and dense distribution ( $\rho_{0}$ and $k_{\text {rep }}$ are large), which has a greater impact on the repulsion function of the artificial potential field, the path curve is smooth, the result just related to the parameters (path is influenced by attraction and repulsion), so that it takes a shorter time to operate(it is unnecessary to search path), and distance off course is not large (airplane fly around volcanic ash clouds), but this causes the airplane to take a long detour, which makes the distance become longer, and the change of angle is larger, so the path planning result is not ideal. The performance of the genetic algorithm is relatively worse, the change of angle is very small, because it rarely allows the aircraft to fly between two ash clouds (in the Madrid-Cairo scenario, the 


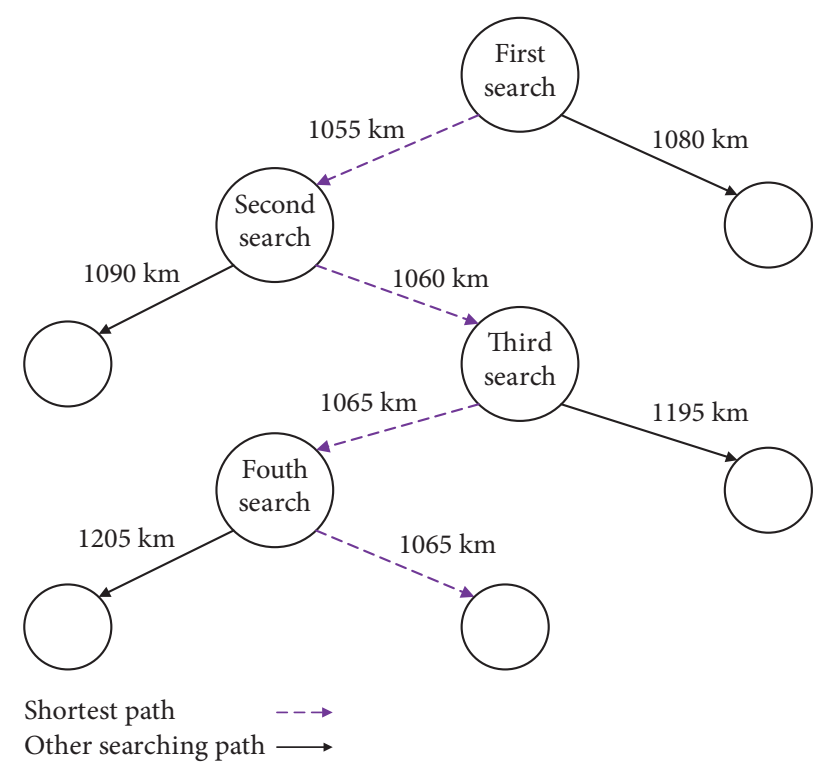

FIgURE 15: Search path $F(n)$ from Algiers to Rome.

\begin{tabular}{|c|c|c|c|c|c|c|c|c|c|c|c|c|c|c|c|c|c|c|}
\hline \multicolumn{19}{|l|}{ Madrid } \\
\hline 1 & 2 & 5 & 10 & 17 & 26 & 35 & 43 & 51 & 62 & 74 & 88 & 105 & 122 & 142 & 162 & 178 & 194 & 213 \\
\hline 3 & 4 & 6 & 11 & 18 & 27 & 36 & 44 & 52 & 63 & 75 & 89 & 106 & 123 & 143 & 163 & 179 & 195 & 214 \\
\hline 7 & 8 & 9 & 12 & 19 & 28 & 37 & 45 & 53 & 64 & 76 & 90 & 107 & 124 & 144 & 164 & 180 & 196 & 215 \\
\hline 13 & 14 & 15 & 16 & 20 & 29 & 38 & 46 & 54 & 65 & 77 & 91 & 108 & 125 & 145 & 165 & 181 & 197 & 216 \\
\hline 21 & 22 & 23 & 24 & 25 & 30 & 39 & & & & & 92 & 109 & 126 & 146 & 166 & 182 & 198 & 217 \\
\hline 31 & 32 & 33 & & & 34 & & & & & & 110 & 111 & 127 & 147 & 167 & 183 & 199 & 218 \\
\hline 40 & 41 & & & & 42 & & & & & & 128 & 129 & 130 & & & & 200 & 219 \\
\hline 47 & 48 & 49 & & & 50 & & & & & & 148 & 149 & & & & & & 220 \\
\hline 55 & 56 & 57 & 58 & 61 & 59 & 60 & & & & & 139 & 158 & & & & & & 238 \\
\hline 66 & 67 & 68 & 69 & 70 & 71 & 72 & 73 & 86 & 102 & 119 & \begin{tabular}{|l|}
137 \\
\end{tabular} & 156 & & & & & & 253 \\
\hline 78 & 79 & 80 & 81 & 82 & 83 & 84 & 85 & 87 & 103 & 120 & 138 & 157 & 174 & & & & & 256 \\
\hline 93 & 94 & 95 & 96 & 97 & 98 & 99 & 100 & 101 & 104 & 121 & 140 & 159 & 175 & 191 & 209 & 230 & 240 & 254 \\
\hline 112 & 113 & 114 & 115 & 116 & 117 & 118 & & & & & 141 & 160 & 176 & 192 & 210 & 231 & 241 & 255 \\
\hline 131 & 132 & 133 & 134 & 135 & 136 & & & & & & & 161 & & & & 232 & 242 & 257 \\
\hline 150 & 151 & 152 & 153 & 154 & 155 & & & & & & & 177 & & & & 243 & 244 & 258 \\
\hline 168 & 169 & 170 & 171 & 172 & 173 & & & & & & & 193 & & & & 259 & 260 & 261 \\
\hline 184 & 185 & 186 & 187 & 188 & 189 & 190 & & & & & & 211 & 212 & 236 & 250 & 262 & 265 & 266 \\
\hline 201 & 202 & 203 & 204 & 205 & 206 & 207 & 208 & & & & 235 & 233 & 234 & 237 & 251 & 263 & 267 & 269 \\
\hline 221 & 222 & 223 & 224 & 225 & 226 & 227 & 228 & 229 & 239 & 249 & 247 & 245 & 246 & 248 & 252 & 264 & 268 & \\
\hline & & & & & & & & & & & & & & & & & & iro \\
\hline
\end{tabular}

Figure 16: The searching process of traditional A-star algorithm from Madrid to Cairo.

plane goes around the southwest side of all the ash clouds), but it also has the longest distance and the longest distance off course. Also, it needs much more time to operate. The shortcomings of premature convergence of the genetic algorithm are found in repeated experiments (optimization based on the initial solution can only reach the local optimal solution). The performance of the traditional A-star algorithm is much better, but the exploration of nodes will have a great impact on the $h(n)$ function. Because the aircraft always chooses the point with the smallest total cost of moving $F(n)$ when exploring the surroundings ( 8 nodes), the path length is shorter. At the same time, the 


\begin{tabular}{|l|l|l|l|l|l|l|l|l|l|l|l|l|l|l|l|l|l|l|l|}
\hline & & & & & & & & & & & & & & & & & & \\
\hline & & & & & & & & & & & & & & & & & \multicolumn{2}{|l|}{ Rome } \\
\hline 83 & 84 & 85 & 86 & 87 & 88 & 89 & 90 & 91 & 97 & 104 & 114 & 124 & 134 & 141 & 142 & 150 & 159 & 171 & \\
\hline 67 & 68 & 69 & 70 & 71 & 72 & 73 & 74 & & & & & 125 & 132 & 133 & & & 160 & 172 & 173 \\
\hline 53 & 54 & 55 & 56 & 57 & 58 & 60 & & & & & & 122 & 123 & & & & & 169 & 170 \\
\hline 40 & 41 & 42 & 43 & 44 & 46 & 59 & & & & & & 111 & 112 & & & & & 157 & 167 \\
\hline 29 & 30 & 31 & 32 & 34 & 45 & 47 & 48 & & & & & 102 & 109 & 119 & & & 147 & 155 & 165 \\
\hline 21 & 22 & 23 & 25 & 33 & 35 & 36 & 39 & 51 & 63 & 77 & 94 & 100 & 107 & 117 & 128 & 137 & 145 & 153 & 163 \\
\hline 14 & 15 & 17 & 24 & 26 & 27 & 28 & 37 & 49 & 61 & 75 & 92 & 98 & 105 & 115 & 126 & 135 & 143 & 151 & 161 \\
\hline 8 & 9 & 16 & 18 & 19 & 20 & & 38 & 50 & 62 & 76 & 93 & 99 & 106 & 116 & 127 & 136 & 144 & 152 & 162 \\
\hline 5 & & & 12 & 13 & & & & 52 & 64 & 78 & 95 & 101 & 108 & 118 & 129 & 138 & 146 & 154 & 164 \\
\hline 3 & & & 7 & 11 & & & & 65 & 66 & 79 & 96 & 103 & 110 & 120 & 130 & 139 & 148 & 156 & 166 \\
\hline 1 & 2 & 4 & 6 & 10 & & & 82 & 80 & 81 & & & & 113 & 121 & 131 & 140 & 149 & 158 & 168 \\
\hline \hline \multicolumn{2}{|c|}{ Algiers } & & & & & & & & & & & & & & & & & & \\
\hline & & & & & & & & & & & & & & & & & & & \\
\hline & & & & & & & & & & & & & & & & & & & \\
\hline & & & & & & & & & & & & & & & & & & \\
\hline
\end{tabular}

FIgURE 17: The searching process of traditional A-star algorithm from Algiers to Rome.

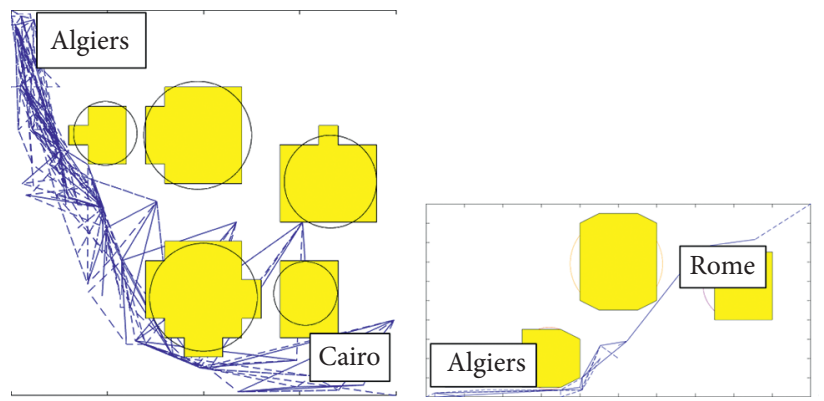

Figure 18: The searching process of genetic algorithm.

aircraft bypasses the volcanic ash cloud, so the deviation distance is shorter. The fixed exploration formula reduces the running time, but the search direction is fixed, which leads to more turns and the largest amount of angle change. ORCA algorithm decreases the search nodes of the A-star algorithm, thereby reducing the complexity; shorter path and smaller turning angles make it easier for pilots to operate; it also reduces the distance off course. In summary, the improved A-star algorithm performs well in both computation time and simulation results, but in some repeated experiments, the results are quite different from the optimal path. The reason may be that the selection of a critical velocity vector has a great influence on the result.

Comparison of improved A-star algorithm and traditional A-star algorithm is shown in Table 8, Figure 21, Table 9, and Figure 22.

The A-star algorithm and genetic algorithm are calculated in a raster map, resulting in a path that is not smooth enough. At the same time, it is difficult to accurately express grids that are not completely covered by obstacles, and it is difficult to express dynamic obstacles. The A-star algorithm has limited exploration directions ( 4 or 8 directions) and lacks flexibility, which causes the airplane to fly in a broken line even in an obstacle-free area. The encoding and decoding process of the genetic algorithm is more complicated, some parameters (population size, crossover rate, and mutation rate) need to rely on empirical selection, the convergence speed is slow, and the calculation time is long. In the artificial potential field method, the moving object is moved by the resultant force, and it is possible that the local resultant force will be zero, and a locally optimal solution will appear. In addition, the aircraft will only avoid obstacles within a certain range from the obstacles, and the avoidance route cannot be planned in advance, causing the aircraft to detour far. The result is affected by the parameters. Improved A-star algorithm to explore the number of nodes is related to the number and density of obstacles that affect the flight of the aircraft, so it will be very complicated when there are 
TABle 6: The results of path planning from Madrid to Cairo.

\begin{tabular}{|c|c|c|c|c|}
\hline Algorithm category & $\begin{array}{l}\text { Improved A-star } \\
\text { algorithm }[23,24]\end{array}$ & $\begin{array}{l}\text { Traditional A-star } \\
\text { algorithm }[14,17]\end{array}$ & $\begin{array}{l}\text { Artificial potential field method } \\
\qquad[12,13]\end{array}$ & Genetic algorithm [10] \\
\hline Path length & $3441 \mathrm{~km}$ & $3653 \mathrm{~km}$ & $3760 \mathrm{~km}$ & $3920 \mathrm{~km}$ \\
\hline Change of angle & $80^{\circ}$ & $360^{\circ}$ & $211^{\circ}$ & $90^{\circ}$ \\
\hline Distance off course & $173 \mathrm{~km}$ & $200 \mathrm{~km}$ & $180 \mathrm{~km}$ & $453 \mathrm{~km}$ \\
\hline Run time & $0.084 \mathrm{~s}$ & $1.770 \mathrm{~s}$ & $0.072123 \mathrm{~s}$ & $353.036 \mathrm{~s}$ \\
\hline $\begin{array}{l}\text { Algorithmic } \\
\text { complexity }\end{array}$ & $O(2 n)$ & $O\left(n^{2}\right)$ & $O\left(n^{2}\right)$ & $O\left((n-1)^{2}\right)$ \\
\hline
\end{tabular}

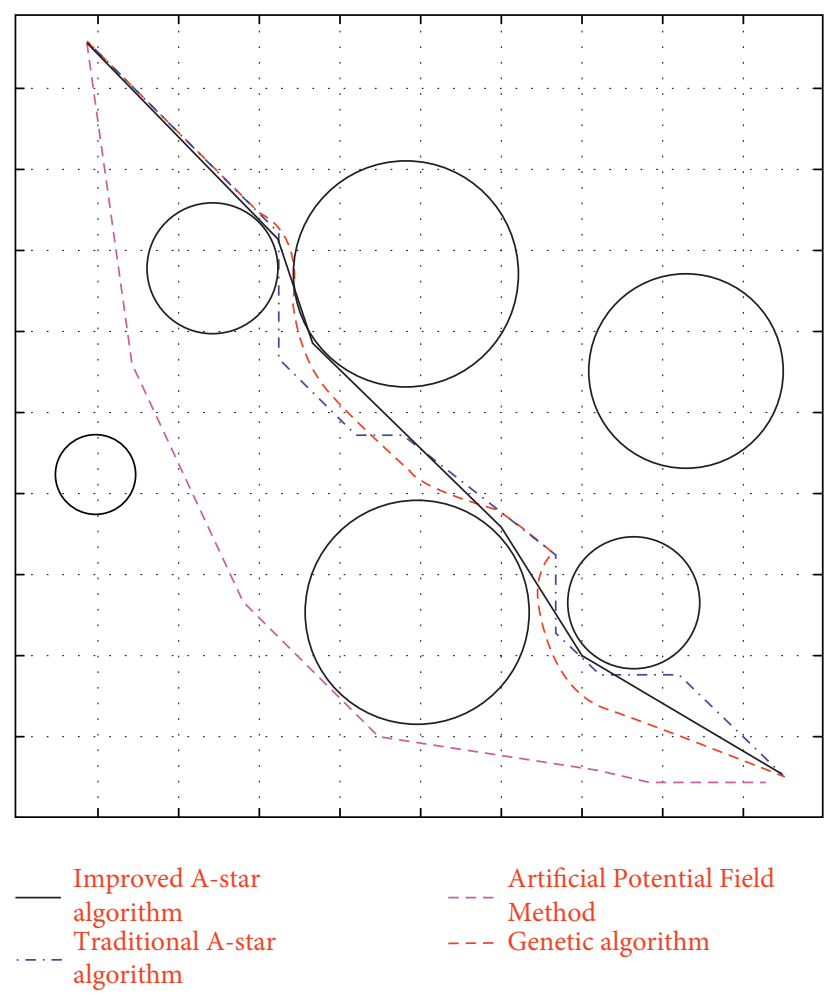

Figure 19: The results of path planning from Madrid to Cairo.

Table 7: The results of path planning from Algiers to Rome.

\begin{tabular}{lcccc}
\hline \multirow{2}{*}{ Algorithm category } & $\begin{array}{c}\text { Improved A-star algorithm } \\
{[23,24]}\end{array}$ & $\begin{array}{c}\text { Traditional A-star algorithm } \\
{[14,17]}\end{array}$ & $\begin{array}{c}\text { Artificial potential field } \\
\text { method [12, 13] }\end{array}$ & Genetic algorithm [10] \\
\hline Path length & $1060 \mathrm{~km}$ & $1155 \mathrm{~km}$ & $1175 \mathrm{~km}$ & $1180 \mathrm{~km}$ \\
Change of angle & $89^{\circ}$ & $270^{\circ}$ & $105^{\circ}$ & $82^{\circ}$ \\
$\begin{array}{l}\text { Distance off course } \\
\text { Run time }\end{array}$ & $45 \mathrm{~km}$ & $130 \mathrm{~km}$ & $155 \mathrm{~km}$ & $160 \mathrm{~km}$ \\
$\begin{array}{l}\text { Algorithmic } \\
\text { complexity }\end{array}$ & $0.030 \mathrm{~s}$ & $1.167 \mathrm{~s}$ & $0.227643 \mathrm{~s}$ & $162.120050 \mathrm{~s}$ \\
\hline
\end{tabular}

many obstacles or densely distributed. The route planned by this algorithm is close to the obstacle, and the safety is poor. Therefore, it is necessary to set a protection zone around the obstacle in advance when planning the route.

Based on the results of static path planning by the improved A-star algorithm, in dynamic path planning, it is assumed that the volcanic ash cloud is an aircraft with a large area, a slow moving speed, and inability to actively avoid obstacles. According to the flight information, it is assumed that the speed of the aircraft is $900 \mathrm{~km} / \mathrm{h}$ (assuming that the speed of the aircraft on the flight path remains unchanged). Since this path is in the prevailing westerly zone with a southwest wind, the movement speed is $10 \mathrm{~m} / \mathrm{s}$ (uniform linear motion), and the wind direction is $45^{\circ}$ (assuming that the movement speed and size of volcanic ash cloud remain unchanged). The results of dynamic path planning from Madrid to Cairo are shown in Table 10 and Figure 23.

The results of dynamic path planning from Algiers to Rome are shown in Table 11 and Figure 24. 


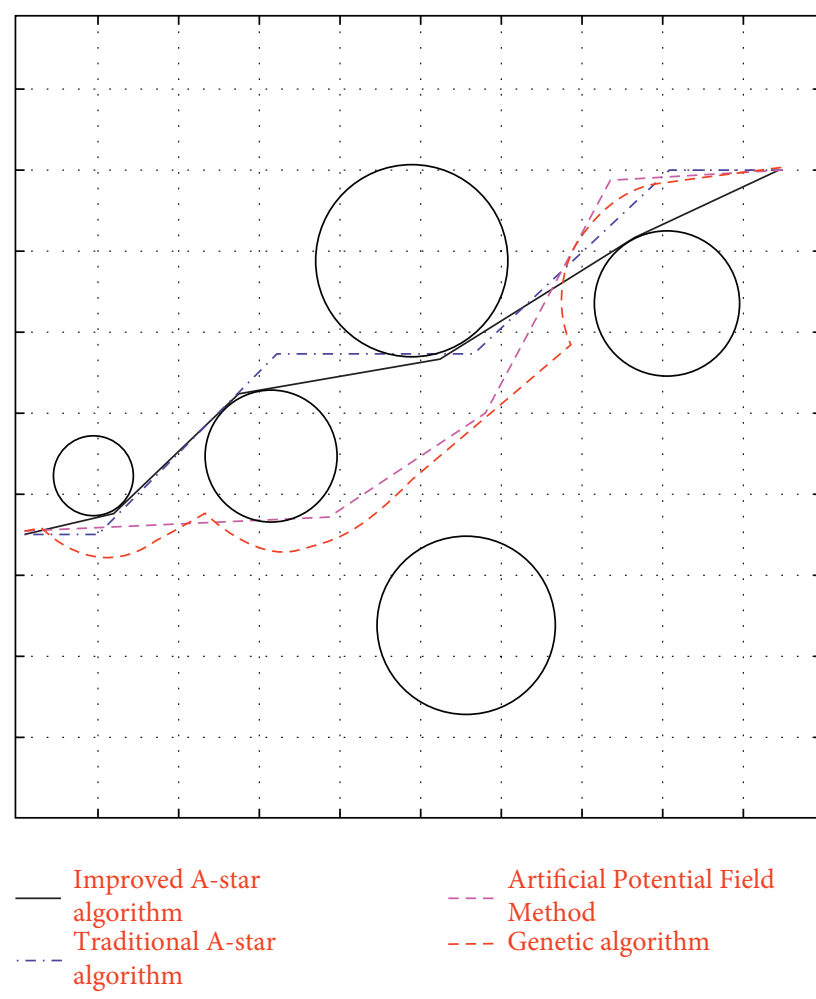

Figure 20: The results of path planning from Algiers to Rome.

TABle 8: Comparison of the improved A-star algorithm and traditional A-star algorithm from Madrid to Cairo.

\begin{tabular}{lccccc}
\hline Item & $\begin{array}{c}\text { Improved A-star } \\
\text { algorithm }\end{array}$ & $\begin{array}{c}\text { Traditional A-star } \\
(10 * 10)\end{array}$ & $\begin{array}{c}\text { Traditional A-star } \\
(20 * 20)\end{array}$ & $\begin{array}{c}\text { Traditional A-star } \\
(30 * 30)\end{array}$ & Traditional A-star (40*40) \\
\hline Search & 2 & & & 8 & \\
directions & 8 & 656 & 2152 & 4744 & 8344 \\
Explore nodes & 8 & 0.717 & 1.770 & 4.518 & 10.446 \\
Run time (s) & 0.084 & & & 8 \\
\hline
\end{tabular}

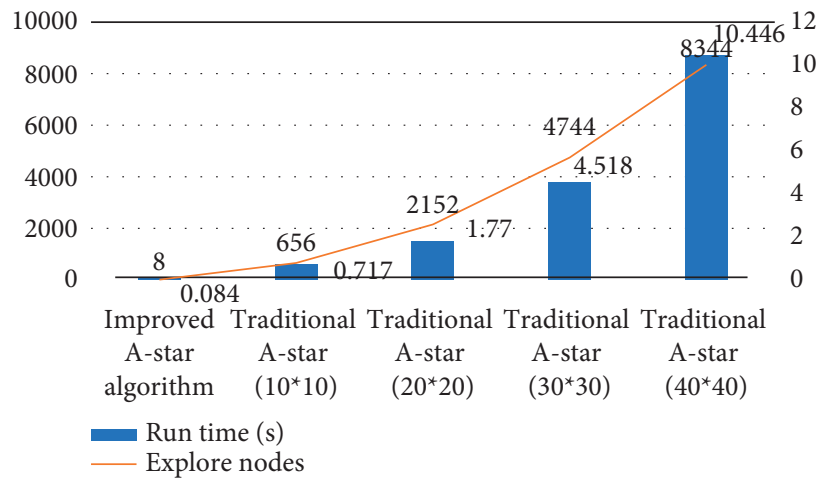

Figure 21: Comparison of the improved A-star algorithm and traditional A-star algorithm from Madrid to Cairo.

TABle 9: Comparison of the improved A-star algorithm and traditional A-star algorithm from Algiers to Rome.

\begin{tabular}{lccccc}
\hline Item & $\begin{array}{c}\text { Improved A-star } \\
\text { algorithm }\end{array}$ & $\begin{array}{c}\text { Traditional A-star } \\
(10 * 10)\end{array}$ & $\begin{array}{c}\text { Traditional A-star } \\
(20 * 20)\end{array}$ & $\begin{array}{c}\text { Traditional A-star } \\
(30 * 30)\end{array}$ & Traditional A-star (40*40) \\
\hline $\begin{array}{l}\text { Search } \\
\text { directions }\end{array}$ & 2 & & & 8 & \\
Explore nodes & 8 & 336 & 1248 & 2872 & 4616 \\
Run time (s) & 0.030 & 0.520 & 1.167 & 2.409 & 4.446 \\
\hline
\end{tabular}




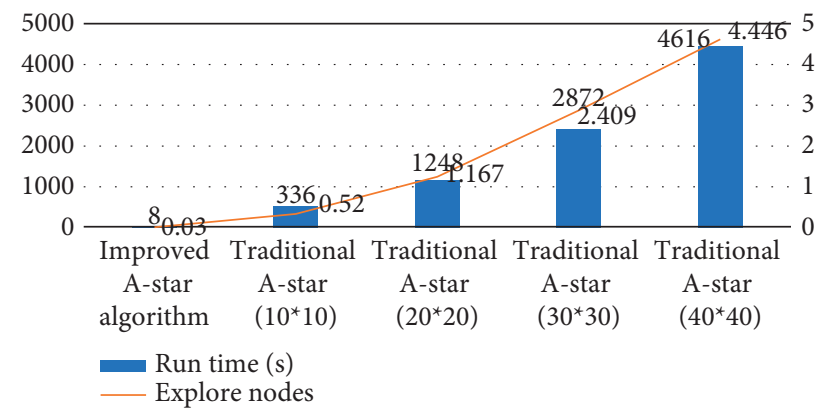

FIgURE 22: Comparison of the improved A-star algorithm and traditional A-star algorithm from Algiers to Rome.

TABLE 10: The results of dynamic path planning from Madrid to Cairo.

\begin{tabular}{lcc}
\hline Items & Static path planning & Dynamic path planning \\
\hline Path length & $3441 \mathrm{~km}$ & $3387 \mathrm{~km}$ \\
Change of angle & $80^{\circ}$ & $74^{\circ}$ \\
Distance off course & $173 \mathrm{~km}$ & $44 \mathrm{~km}$ \\
\hline
\end{tabular}

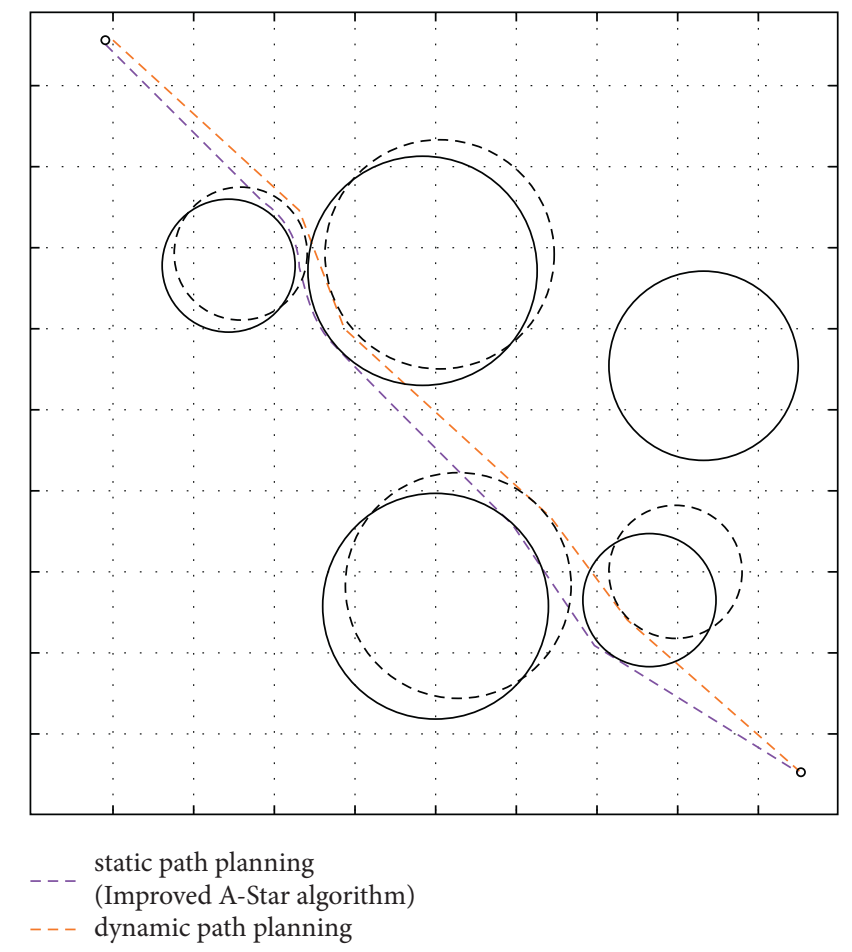

FIGURE 23: The results of dynamic path planning from Madrid to Cairo.

TABLE 11: The results of dynamic path planning from Algiers to Rome.

\begin{tabular}{lcc}
\hline Items & Static path planning & Dynamic path planning \\
\hline Path length & $1060 \mathrm{~km}$ & $1063 \mathrm{~km}$ \\
Change of angle & $89^{\circ}$ & $82^{\circ}$ \\
Distance off course & $45 \mathrm{~km}$ & $50 \mathrm{~km}$ \\
\hline
\end{tabular}

Since the ash cloud is moving much slower than the speed of the aircraft, the impact on the path length is small, in terms of path length, the first scenario increased by $-1.54 \%$, and the second scenario decreased by $0.28 \%$. Also, the influence of distance off course is small. However, the aircraft may approach other ash clouds while avoiding dynamic ash clouds. If the ash cloud moves at a high speed, the aircraft may not be able to bypass other ash clouds, and the path needs to be replanned. 


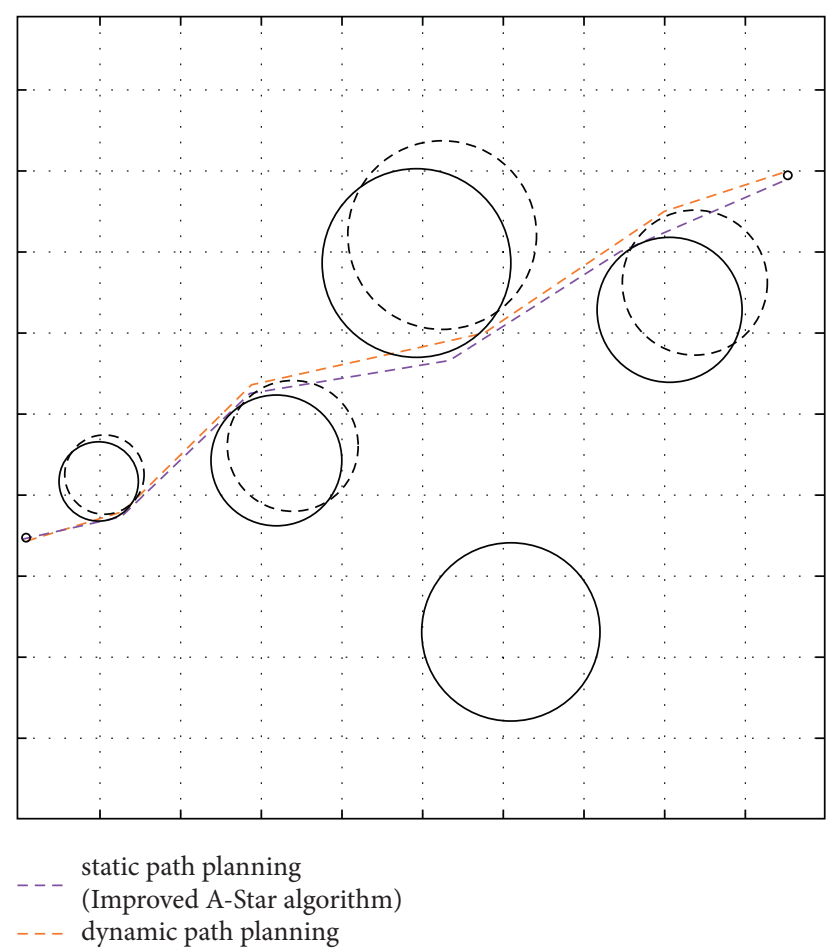

FIgure 24: The results of dynamic path planning from Algiers to Rome.

\section{Conclusions}

This paper plans the path in the volcanic ash area and uses the speed vector idea of the ORCA algorithm to improve the A-star algorithm. By introducing the concept of the potential collision set, the speed and acceleration set of the aircraft in the volcanic ash area are obtained. We select the optimal speed range of the aircraft from the limited elements, calculate the flight trajectory by accumulating the displacement increments, and continuously update the $F(n)$ value to obtain the final path planning result. We solve the problems of traditional A-star algorithm in aircraft flight path planning, such as many search nodes, wide search directions, and long computing time. The main research work is reflected as follows. First, we establish an algorithm model for the volcanic ash area. Based on the algorithm model, we select a reasonable speed range for the aircraft. Second, we improve the path planning algorithm to complete the pathfinding. We draw lessons from the speed vector idea of the ORCA algorithm and use a critical speed vector for path planning to optimize the underlying structure of the A-star algorithm. Third, we introduce the evaluation function. The distance between the airplane and the volcanic ash area is weighted, and the speed and direction of the airplane are weighted. The two together constitute the evaluation function update $h(n)$ function and $F(n)$ function.

The algorithm used in this paper is an improvement in the exploration direction of the A-star algorithm. The planning result is a global optimal solution and will not fall into the local optimal problem. The performance of the genetic algorithm is relatively good, but the shortcomings of premature convergence of genetic algorithms are found in repeated experiments. The performance of the traditional A-star algorithm is unstable, and the exploration of nodes will have a great impact on the $h(n)$ function. However, the algorithm does not involve the aspect of reducing exploration nodes. You can start from this aspect to reduce computing time and improve algorithm efficiency.

In the two experimental scenarios, the improved A-star algorithm has advantages in terms of path length, the distance between aircraft and volcanic ash area, distance off course, and computational complexity, which is generally superior to other algorithms. Compared with static path planning, dynamic path planning reduces the distance between the aircraft and the ash area, and the path length remains basically unchanged.

This paper plans the route in the volcanic ash area and uses the speed vector idea of the ORCA algorithm to improve the A-star algorithm.

By introducing the concept of a potential collision set, the speed and acceleration set of the aircraft in the volcanic ash area is obtained. The optimal speed range of the aircraft is selected from the finite elements, the flight trajectory is calculated by the cumulative displacement increment, and the $F(\mathrm{n})$ value is continuously updated to obtain the final path planning result. The traditional A-star algorithm solves the problems of multiple search nodes, wide search directions, and long calculation time in aircraft flight path planning. The main research work is as follows. First, we establish an algorithm model for the volcanic ash area. According to the algorithm model, we select a reasonable speed range for the aircraft. Second, we improve the path planning algorithm to complete the path search. Using the speed vector idea of the ORCA algorithm for reference, the 
critical speed vector is used for path planning, and the underlying structure of the A-star algorithm is optimized. Finally, the evaluation function is introduced. The distance between the aircraft and the volcanic ash area is weighted, and the speed and direction of the aircraft are weighted. The two together constitute the evaluation function update $h(\mathrm{n})$ function and $F(n)$ function.

In future research, we can try to add restriction conditions in the loop statement of the algorithm to achieve the purpose of identifying the exploration node. We will try our best to avoid obstacles and avoid conflicts with other flights by climbing, descending, or changing speed. We will also consider the expansion or contraction of the ash area while increasing the evaluation indicators such as fuel consumption and flight time.

\section{Data Availability}

No data were used to support the study.

\section{Conflicts of Interest}

The authors declare that they have no conflicts of interest.

\section{References}

[1] Yi Zhao, Y. Li, and Q. Fan, "The progress of volcanic ash cloud study in aviation safety," Bulletin of Mineralogy, Petrology and Geochemistry, vol. 33, no. 04, pp. 531-539, 2014.

[2] P. Zhang, Research on Global Dynamic Path Planning Based on Improved A* Algorithm, North University of China, Taiyuan, China, 2021.

[3] D. Myers, R. Batta, and M. Karwan, "A real-time network approach for including obstacles and flight dynamics in UAV route planning," The Journal of Defense Modeling and Simulation, vol. 13, no. 3, pp. 291-306, 2016.

[4] J. C. H. Cheung, "Flight planning: node-based trajectory prediction and turbulence avoidance," Meteorological Applications, vol. 25, no. 1, pp. 78-85, 2017.

[5] B. K. Patle, D. R. K. Parhi, A. Jagadeesh, and S. K. Kashyap, "Matrix-Binary Codes based Genetic Algorithm for path planning of mobile robot," Computers \& Electrical Engineering, vol. 67, pp. 708-728, 2018.

[6] E. D’Amato, M. Mattei, and I. Notaro, "Bi-level flight path planning of UAV formations with collision avoidance," Journal of Intelligent and Robotic Systems, vol. 93, 2018.

[7] M. Zhang, X. Kong, K. Liu, and X Li, "A novel rerouting planning model for the terminal arrival routes under the influence of convective weather," Journal of Advanced Transportation, vol. 2018, no. 2, 21 pages, Article ID 7591932, 2018.

[8] D. Li, "Analysis on route planning of flight diversion under dangerous weather," Military and civilian technology and products, vol. 2016, no. 4, p. 5, 2016.

[9] Q. Wang, D. Yu, and Y. Jia, "The A-star: an any-angle path planning algorithm based on terrain risk," Journal of Deep Space Exploration, vol. 1, no. 4, pp. 269-274, 2014.

[10] Y. Wang, Y. Zheng, D. Yao, L. Zhengxin, and L. Chao, "Rerouting path network planning under dangerous weather," Systems Engineering and Electronics, vol. 41, no. 6, pp. 1309-1315, 2019.

[11] A. Zhang, S. Ma, B. Li, M. Wang, and J. Chang, "Parameter optimization of eel robot based on NSGA-II algorithm," in
Proceedings of the 12th International Conference on Intelligent Robotics and Applications (ICIRA 2019), pp. 3-15, Shenyang, China, August 2019.

[12] U. Orozco-Rosas, O. Montiel, and R. Sepúlveda, "Mobile robot path planning using membrane evolutionary artificial potential field," Applied Soft Computing, vol. 77, pp. 236-251, 2019.

[13] U. Orozco-Rosas, K. Picos, and O. Montiel, "Hybrid path planning algorithm based on membrane pseudo-bacterial potential field for autonomous mobile robots," IEEE Access, vol. 7, pp. 156787-156803, 2019.

[14] Y. Zheng, Y. Wang, and M. Lv, "Route planning and design research based on improved A-star algorithm [J]," Fire Control and Command Control, vol. 43, no. 9, pp. 125-129, 2018.

[15] H. Bast, D. Delling, A. Goldberg et al., Route Planning in Transportation Networks, Springer International Publishing, New York, NY, USA, 2016.

[16] Y. Zhou, X. Cheng, X. Lou, Z Fang, and J Ren, "Intelligent Travel Planning System Based on A-star Algorithm," in Proceedings of the 2020 IEEE 4th Information Technology, Networking, Electronic and Automation Control Conference, Chongqing, China, June 2020.

[17] G. Chen, T. Wu, and Z. Zhou, "Research on ship meteorological route based on A-star algorithm," Mathematical Problems in Engineering, vol. 2021, no. 7, 8 pages, Article ID 9989731, 2021.

[18] J. van den Berg, S. J. Guy, M. Lin, and D. Manocha, "Reciprocal n-body collision avoidance," Springer Tracts in Advanced Robotics, vol. 70, pp. 3-19, 2011.

[19] H. Zhao, Design of Dynamic Route Planning Method Based on Live Weather, Harbin Engineering University, Harbin, China, 2017.

[20] V. Singh and K. E. Willcox, "Methodology for path planning with dynamic data-driven flight capability estimation," AIAA Journal, vol. 55, no. 8, pp. 1-12, 2016.

[21] A. I. Croce, G. Musolino, C. Rindone, and A vitetta, "Route and path choices of freight vehicles: a case study with floating car data," Sustainability, vol. 12, no. 20, pp. 1-15, 2020.

[22] G. Musolino, C. Rindone, and A. Vitetta, "A modelling framework to simulate paths and routes choices of freight vehicles in sub-urban areas," in Proceedings of the 7th International Conference on Models and Technologies for Intelligent Transportation Systems (MT-ITS), Heraklion, Greece, June 2021.

[23] J. Chen, C. Du, Y. Zhang, P. Han, and W. Wei, "A clusteringbased coverage path planning method for autonomous heterogeneous UAVs," IEEE Transactions on Intelligent Transportation Systems, pp. 1-11, 2021.

[24] W. Zhou, X. Wang, H. Sun, and Y. Chen, "Trajectory optimization of quadrotor UAV based on Bessel curve," Journal of Electronic Measurement and Instrumentation, vol. 33, no. 10, pp. 53-58, 2019.

[25] J. Chen, M. Li, Z. Yuan, and Q. Gu, "An Improved A* Algorithm for UAV Path Planning Problems," in Proceedings of the 2020 IEEE 4th Information Technology, Networking, Electronic and Automation Control Conference (ITNEC), Chongqing, China, May 2020.

[26] F. Ling, J. Chen, and C. Du, "Multi-obstacle path planning of UAV based on Improved Ant Colony System Algorithm," in Proceedings of the 2020 IEEE 5th Information Technology and Mechatronics Engineering Conference (ITOEC), Chongqing, China, July 2020. 
[27] S. Yang and X. Zhang, "Energy optimized maneuver trajectory generation for unmanned aerial vehicles[J]," Acta Aeronautica et Astronautica Sinica, vol. 41, no. S2, Article ID 724288, 2020.

[28] Wu Deng, Research and Application of Agents Obstacle Avoidance and Path Planning Based on Deep Reinforcement Learning, University of Electronic Science and Technology of China, Chengdu, China, 2019.

[29] C. Zu, The Parallel Driving System Based on Improved Multiple Vehicles Collision Avoidance Algorithms, Jilin University, Changchun, China, 2019. 